\title{
过渡金属催化 $\mathrm{P}-\mathrm{C}$ 键偶联反应的研究进展
}

\author{
郡长伟徐炜刚李 亮* 张兴华*
}

(上海应用技术大学化学与环境工程学院 上海 201418)

\begin{abstract}
摘要 含有 $\mathrm{P}-\mathrm{C}$ 键的有机膦化合物在光电、阻燃材料以及药物化学等研究领域具有广泛的应用. 通过过渡金属催化 $\mathrm{P}(\mathrm{O})-\mathrm{H}$ 化合物的交叉偶联构建 $\mathrm{P}-\mathrm{C}$ 键是合成功能有机膦化合物的重要方法. 按碳原子的不同杂化类型分类，对此类 反应的研究进展进行了综述.
\end{abstract}

关键词 过渡金属催化; $\mathrm{P}-\mathrm{C}$ 键；交叉偶联

\section{Recent Advances of Transition Metal-Catalyzed P-C Coupling Reactions}

\author{
Shao, Changwei Xu, Weigang Li, Liang* Zhang, Xinghua* \\ (School of Chemical and Environmental Engineering, Shanghai Institute of Technology, Shanghai 201418)
}

\begin{abstract}
Organophosphorus compounds which contain $\mathrm{P}-\mathrm{C}$ bonds have been widely used in photoelectric materials, retardant materials and medicinal chemistry. It is an important method for the synthesis of functional organophosphorus compounds from $\mathrm{P}(\mathrm{O})-\mathrm{H}$ reagents using transition metal-catalyzed cross coupling reaction to form $\mathrm{P}-\mathrm{C}$ bond. The recent development in this area is summarized on the basis of different types of carbon atom.
\end{abstract}

Keywords transition metal-catalyzed; $\mathrm{P}-\mathrm{C}$ bond; cross-coupling

含有磷碳 $(\mathrm{P}-\mathrm{C})$ 键的有机膦化合物在有机合成、催 化、功能材料以及药物化学等领域具有广泛的应用 ${ }^{[1 \sim 5]}$, 并展现出许多优异性能. 由于 $\mathrm{P}-\mathrm{C}$ 键特殊的化学性质, 含有膦酸基团 $\left[\mathrm{R}-\mathrm{PO}(\mathrm{OH})_{2}\right]$ 结构的药物被广泛用于糖 尿病、心力衰竭、症疾、艾滋病等 ${ }^{[6 ~ 10]}$ 的治疗(图 1). 天 然产物中也存在很多含有 $\mathrm{P}-\mathrm{C}$ 键的有机膦化合物 ${ }^{[1]}$, 如 Bialaphos 和 Fosfomycin 就已经商业化, 并作为除草 剂和抗生素被广泛使用.

功能材料方面, 目前研究比较广泛的含磷有机阻燃 剂, 因其无毒无害且具有良好的阻燃效果, 已经得到了 世界范围的公认. 分子中所含有的 $\mathrm{P}-\mathrm{C}$ 键使之具有较 高的热稳定性, 而 $\mathrm{P}=\mathrm{O}$ 键的存在也使得五价氧膦化合 物(Phosphine Oxide)的抗氧化性能明显高于三价有机膦 化合物(Phosphine).

由此可见, 含有 $\mathrm{P}-\mathrm{C}$ 键的有机膦化合物在有机合 成以及新材料等的研发中具有广泛应用. 如何通过简 单、高效、低毒的方法构建这一类化合物, 尤其是构建<smiles>CCc1cc(F)c(N)c2nc(-c3ccc(P(=O)(O)O)o3)n(CC(C)C)c12</smiles><smiles>O=CN(O)CCC(c1ccc(Cl)c(Cl)c1)P(=O)(O)O</smiles><smiles>CC(Cn1cnc2c(N)ncnc21)OCP(=O)(O)O</smiles><smiles>Nc1nc(Cl)nc2c1ncn2[C@@H]1[C@H](O)[C@H](O)[C@]2(CP(=O)(O)O)C[C@H]12</smiles>

图 1 含磷碳键的药物

Figure 1 Drugs containing $\mathrm{P}-\mathrm{C}$ bonds

具有高度官能化的膦酸酯以及氧膦类化合物，已成为近 年来有机磷化学家研究的热点.

早期构建 $\mathrm{P}-\mathrm{C}$ 键的方法主要是通过 Arbuzov 或类 Arbuzov 反应来实现的(Scheme 1). 该方法使用三配位

\footnotetext{
* Corresponding authors. E-mail: xhzhang@sit.edu.cn; lilianglcx@sit.edu.cn

Received August 29, 2016; revised September 26, 2016; published online October 11, 2016.

Project supported by the National Natural Science Foundation of China (Nos. 21302127, 21502116).

国家自然科学基金(Nos. 21302127, 21502116)资助项目.
} 
磷化合物与烷基卤代物反应, 反应的原子经济性较差 (需消除一分子的卤代烷烃), 并且反应底物比较单一, 尤其是在构建具有特殊结构的有机膦化合物时, 此类方 法不能满足实际的需要. 采用格氏试剂或者锂试剂与氯 代磷(膦)酸酯反应的方法也可构建 $\mathrm{P}-\mathrm{C}$ 键, 但该方法 所使用的氯代磷试剂不稳定, 并且有机金属试剂对水和 氧气比较敏感, 不利于广泛推广.

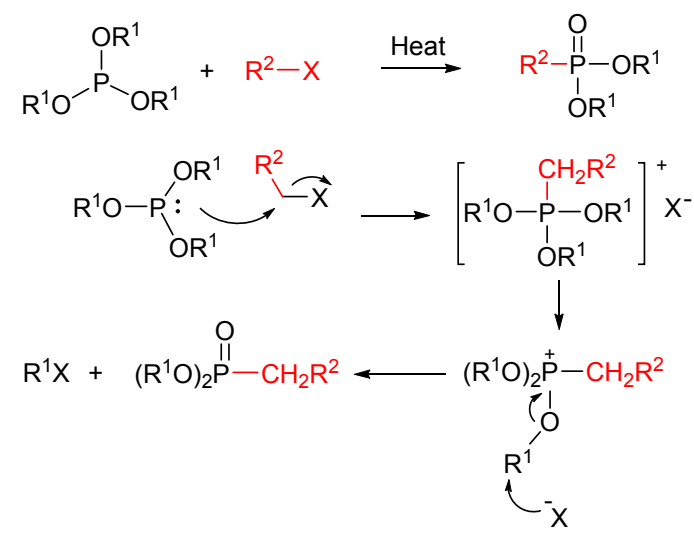

图式 1 Michaelis-Arbuzov 反应及机理

Scheme 1 A plausible mechanism for the reaction of Michaelis-Arbuzov

采用过渡金属催化的方法进行 $\mathrm{P}-\mathrm{C}$ 键的构 建 ${ }^{[12 \sim 14]}$, 因其具有高效、高选择性等特点而成为有机磷 化学研究比较活跃的领域, 目前主要集中在过渡金属 $\mathrm{Pd} 、 \mathrm{Cu} 、 \mathrm{Ni}$ 的催化研究.

\section{1 过渡金属催化构建 $\mathrm{P}-\mathrm{C}\left(\mathrm{sp}^{3}\right)$ 键}

如前所述, 传统构建 $\mathrm{P}-\mathrm{C}\left(\mathrm{sp}^{3}\right)$ 键的方法主要依赖 Michaelis-Arbuzov、Michaelis-Becker 或类 Arbuzov 反应, 但高温及强碱条件大大限制了此类反应的进一步应用. 近期对 Michaelis-Arbuzov 反应的改进取得了很好的进 展，2011 年 Mohanakrishnan 等 ${ }^{[15 a]}$ 首次报道了室温条件 下 Lewis 酸催化的 Michaelis-Arbuzov 反应，从三配位磷 出发, 以苄卤、苄醇、杂环茮卤、杂环茮醇为原料, 在 $\mathrm{ZnBr}_{2}$ 催化下制备了相应的膦酸酯(Eq. 1). 2011 年, Wiemer 等 ${ }^{[15 b]}$ 对此类反应底物进行拓展, 对于含不同取 代基的茮醇或烯丙醇, 在 Lewis 酸 $\mathrm{ZnI}_{2}$ 的催化下均可与 亚磷酸三乙酯反应生成苄基或烯丙基膦酸酯, 收率 $15 \% \sim 85 \%$ (Eq. 2).

近年来, 从 $\mathrm{P}(\mathrm{O})-\mathrm{H}$ 化合物出发构建 $\mathrm{P}-\mathrm{C}$ 键的方 法也受到广泛关注, 并得到不断深入的研究. 2003 年, Salvatore 等 ${ }^{[16]}$ 发展了一种便捷合成膦酸酯的方法, 室温 条件下以 $\mathrm{Cs}_{2} \mathrm{CO}_{3}$ 作碱, 即可实现从 $\mathrm{P}(\mathrm{O})-\mathrm{H}$ 化合物到 $\mathrm{P}-\mathrm{C}\left(\mathrm{sp}^{3}\right)$ 键转变, 但反应底物大多适用于 $1^{\circ}$ 卤代烷烃 以及芐基卤代化合物，具有一定的局限性(Eq. 3). 2009

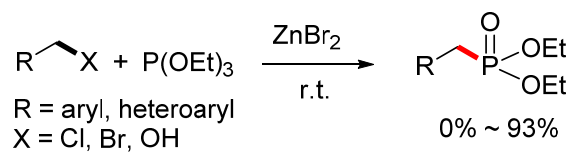

$$
\begin{aligned}
& \underbrace{\mathrm{Znl}_{2}, \mathrm{P}(\mathrm{OEt})_{3}}_{\mathrm{OH}}
\end{aligned}
$$

年, Stawinski 等 ${ }^{[17]}$ 报道了醋酸钯催化下苄卤与氢亚磷酸 酯构建 $\mathrm{P}-\mathrm{C}\left(\mathrm{sp}^{3}\right)$ 键的方法(Eq. 4), 同样存在底物适用性 不强等问题(仅适用于茮基卤代物).

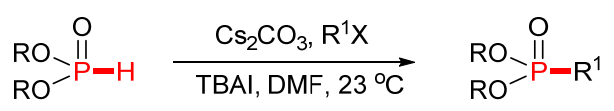

$$
\begin{aligned}
& >_{\mathrm{RO}^{-P}}^{\mathrm{RO}-\mathrm{P}-\mathrm{H}} \\
& \mathrm{X}=\mathrm{Br}, \mathrm{Cl} ; \mathrm{Y}=\mathrm{H}, \mathrm{F}, \mathrm{Cl}, \mathrm{Me} \text {, OMe; } \\
& \mathrm{R}=\text { Alkyl, Aryl, a nucleoside moiety }
\end{aligned}
$$

过渡金属催化 $\mathrm{P}(\mathrm{O})-\mathrm{H}$ 化合物的交叉脱氢偶联反 应(Cross-Dehydrogenative-Coupling, CDC)也是构建 P$\mathrm{C}\left(\mathrm{sp}^{3}\right)$ 键的主要方法. 2009 年, 李朝军等 ${ }^{[18]}$ 以 $\mathrm{CuBr}$ 盐为 催化剂, 氧气作为氧化剂实现了 $\mathrm{P}(\mathrm{O})-\mathrm{H}$ 化合物与 $\mathrm{C}\left(\mathrm{sp}^{3}\right)-\mathrm{H}$ 键 (邻位为氮原子)的脱氢氧化偶联反应, 该 方法在较温和的条件下即可合成具有生物活性的 $\alpha$-氨 基膦酸酯(Eq. 5). 同样, Ofial 等 ${ }^{[19]}$ 也报道了一种二价铁 盐催化氢亚磷酸酯经脱氢氧化制备 $\mathrm{P}-\mathrm{C}\left(\mathrm{sp}^{3}\right)$ 键化合物 的新方法，该方法以过氧化叔丁醇为氧化剂，10 $\mathrm{mol} \%$ 氯化亚铁催化下高效制备了 $\alpha$-氨基膦酸酯类化合物(Eq. 6), 反应的副产物为水.
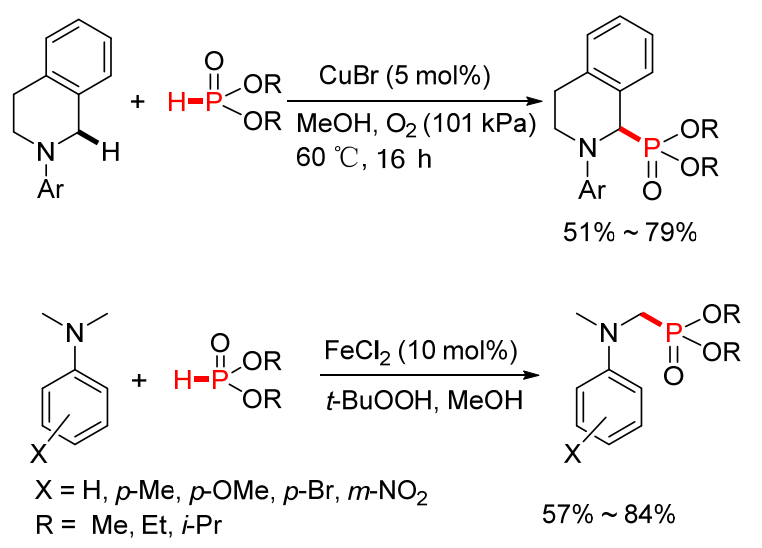

由此可见, 金属 $\mathrm{Cu} 、 \mathrm{Fe}$ 催化 $\mathrm{P}(\mathrm{O})-\mathrm{H}$ 键的脱氢氧 化偶联反应实现了 $\mathrm{P}-\mathrm{C}\left(\mathrm{sp}^{3}\right)$ 键化合物的绿色构建, 也 拓展了合成 $\alpha$-氨基膦酸酯类化合物的新方法. 在此基础 
上杨尚东等 ${ }^{[20]}$ 以 $\mathrm{Ag}_{2} \mathrm{CO}_{3}$ 为氧化剂, 在手性质子酸诱导 下进一步实现了 $\alpha$-氨基膦酸酯类化合物的不对称合成 (Eq. 7), 该反应可适用于含有不同取代基团的烯丙胺及 $\mathrm{P}-\mathrm{H}$ 化合物, 拓展了氧化脱氢偶联反应的底物适用范 围, 并且取得了 $e e$ 值高达 $92 \%$ 的手性选择.

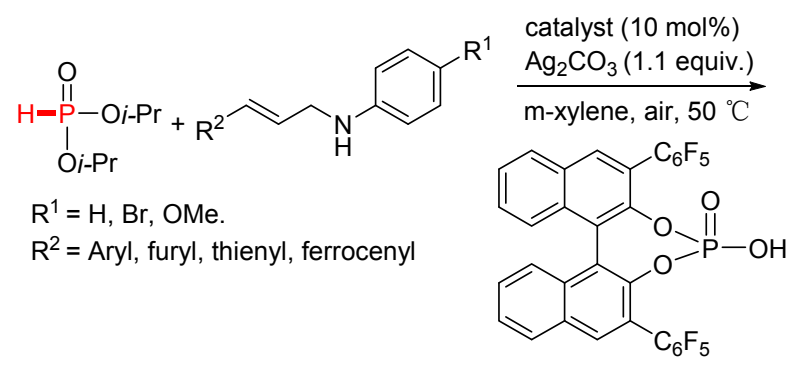<smiles>[R]C=C[C@H](Nc1ccc([R])cc1)P(=O)(O[Tl])OCC</smiles>

up to $e e 92 \%$

2015 年，赵玉芬等 ${ }^{[21]}$ 采用相同的氧化脱氢偶联策 略, 以 $\mathrm{CuSO}_{4} \cdot 5 \mathrm{H}_{2} \mathrm{O}$ 为催化剂, 过氧化叔丁醇( TBHP)为 氧化剂, 通过脱羧氧化偶联实现了由炔基羧酸到 $\beta$-羰基 膦氧化合物的转化. 该反应可在较温和条件下快速、高 效地制备 $\beta$-酩类含磷衍生物, 这也为氧化脱羧偶联构建 $\mathrm{P}-\mathrm{C}\left(\mathrm{sp}^{3}\right)$ 键提供了新的途径(Eq. 8).

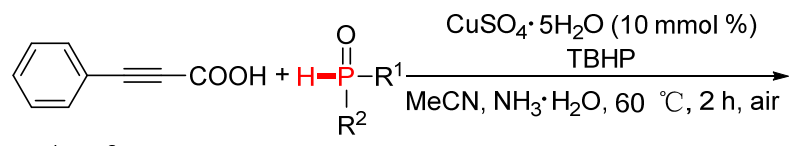

$\mathrm{R}^{1}=\mathrm{R}^{2}=\mathrm{Ph}, \mathrm{Ar}$<smiles>[R]P([R])(=O)CC(=O)c1ccccc1</smiles>

可见光作为新型绿色能源, 在催化构建 $\mathrm{P}-\mathrm{C}\left(\mathrm{sp}^{3}\right)$ 键方面也得到了很好的利用. 2011 年, Koenigs 等 ${ }^{[22 a]}$ 首 次报道了光照条件下, 利用 $R u(I I)$ 或 $I r(I I I)$ 作催化剂, 通 过氧化脱氢高效制备 $\alpha$-氨基膦酸酯, 开辟了可见光催化 构建 $\mathrm{P}-\mathrm{C}\left(\mathrm{sp}^{3}\right)$ 键的新领域(Eq. 9). 通过对催化剂的篎 选，此类反应得到进一步深入的研究，曙红 Y (eosinY)、

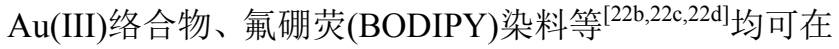
相应光源照射下实现 $\mathrm{P}-\mathrm{C}\left(\mathrm{sp}^{3}\right)$ 键的转化(Eqs. 10, 11, 12). 此外, Rueping 等 ${ }^{[22 \mathrm{e}]}$ 也报道了金属氧化物同样可以 在光照条件下高产率合成 $\alpha$-氨基膦酸酯(Eq. 13), 并且, 金属氧化物具有可循环利用、价格低廉等优点, 具有很 好的应用前景.

近年来, 金属锰盐在实现 $\mathrm{P}-\mathrm{C}\left(\mathrm{sp}^{3}\right)$ 键的转化中也

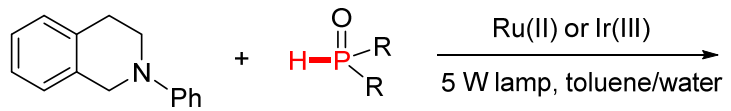

$\mathrm{R}=$ alkyl, aryl, alkoxy<smiles>[R]P([R])(=O)C1c2ccccc2CCN1Pc1ccccc1</smiles>
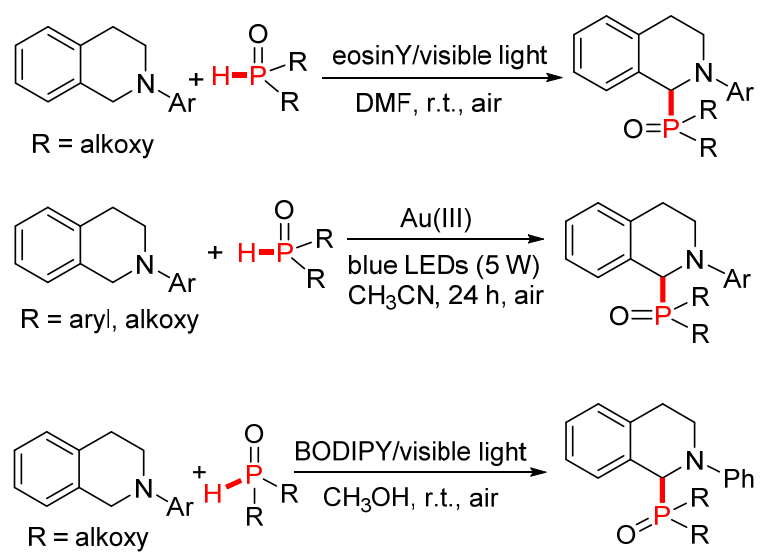

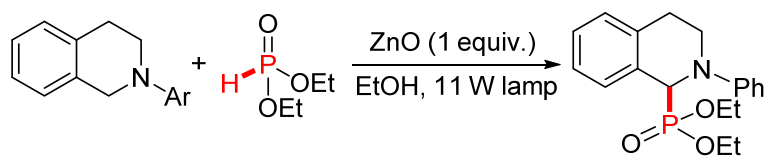

有突出表现. 2015 年, 赵玉芬课题组 ${ }^{[23 a]}$ 报道了系列锰盐 催化构建 $\mathrm{P}-\mathrm{C}\left(\mathrm{sp}^{3}\right)$ 键的新方法. 在 2.5 equiv.二水合醋 酸锰的促进下，以廉价的端烯、 $\mathrm{TMSN}_{3}$ 、亚磷酸酯为原 料，温和条件下即可合成 $\beta$-叠氮膦酸酯，产率 $28 \%$ $88 \%$ (Eq. 14). 同样以锰盐为催化剂 ${ }^{[23 b]}$ ，以不饱和烯酸 为原料, 通过与 $\mathrm{P}(\mathrm{O})-\mathrm{H}$ 试剂偶联的同时也生成了五、 六元环内酯含磷衍生物, 产率中等以上. 该反应具有官 能团耐受性好以及反应条件温和等特点(Eq. 15).

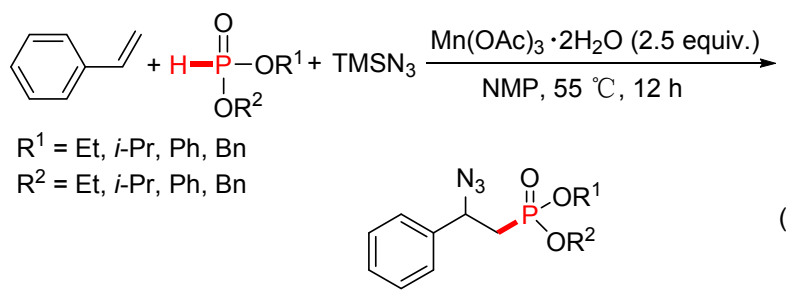

$$
\begin{aligned}
& \mathrm{HOOCC}_{\mathrm{R}} \\
& \mathrm{R}^{1}=\mathrm{R}^{2}=\mathrm{OMe}, \mathrm{OEt}, \mathrm{O}-\mathrm{i}-\mathrm{Pr}, \mathrm{Ph} \\
& \mathrm{R}^{3}=\mathrm{Me}, \mathrm{Ph}, \mathrm{Ar} ; \mathrm{R}^{4}=\mathrm{H}, \mathrm{Ph} ; n=1,2
\end{aligned}
$$

2016 年, 赵玉芬课题组 ${ }^{[24]}$ 实现了 $\mathrm{Cu}(\mathrm{OTf})_{2}$ 催化 $\mathrm{P}(\mathrm{O})-\mathrm{H}$ 试剂与端烯类化合物的环化反应, 并成功合成 
了异喹啉二酮含磷衍生物. 实验表明, $\mathrm{Cu}(\mathrm{II}) / \mathrm{TBHP}$ 协同 作用下所产生的磷自由基是促进反应的关键中间体，通 过改变 $\mathrm{P}(\mathrm{O})-\mathrm{H}$ 试剂的种类, 可以在温和条件下制备不 同类型的含磷异喹啉二酮, 并得到中等以上的收率, 这 也为 $\mathrm{P}-\mathrm{C}\left(\mathrm{sp}^{3}\right)$ 键构建提供了新的途径(Eq. 16).

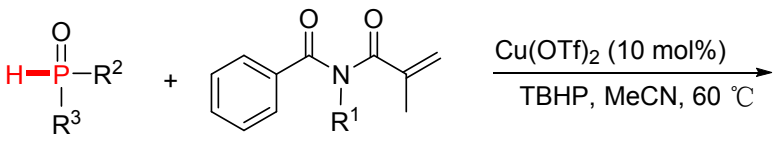<smiles>[R1]N1C(=O)c2ccccc2C(C)(CP([R])([R])=O)C1=O</smiles>

$\mathrm{R}^{1}=\mathrm{Me}, \mathrm{Et}, i-\mathrm{Pr}$

$\mathrm{R}^{2}=\mathrm{OMe}, \mathrm{OEt}, \mathrm{O}-i-\mathrm{Pr} ; \mathrm{R}^{2}=\mathrm{OMe}, \mathrm{OEt}, \mathrm{O}-i-\mathrm{Pr}, \mathrm{Ph}$

\section{2 过渡金属催化构建 $\mathrm{P}-\mathrm{C}\left(\mathrm{sp}^{2}\right)$ 键}

近年来，随着过渡金属催化构建 $\mathrm{C}-\mathrm{X}(\mathrm{X}=\mathrm{C}, \mathrm{N}, \mathrm{S}$, $\mathrm{Si}, \mathrm{B})$ 键的研究深入, 金属催化领域所取得的突破性研 究成果显示了其与传统合成方法相比所具有的优势, 因 此, 采用简单金属催化 $\mathrm{P}-\mathrm{C}\left(\mathrm{sp}^{2}\right)$ 键的合成方法, 因其高 效、高选择性等特点越来越引起人们的关注 ${ }^{[25 ~ 27]}$. 其中, 过渡金属 $\mathrm{Pd} 、 \mathrm{Cu} 、 \mathrm{Ni}$ 对催化实现 $\mathrm{P}-\mathrm{C}\left(\mathrm{sp}^{2}\right)$ 的构建具有 广泛的应用前景(Scheme 2).

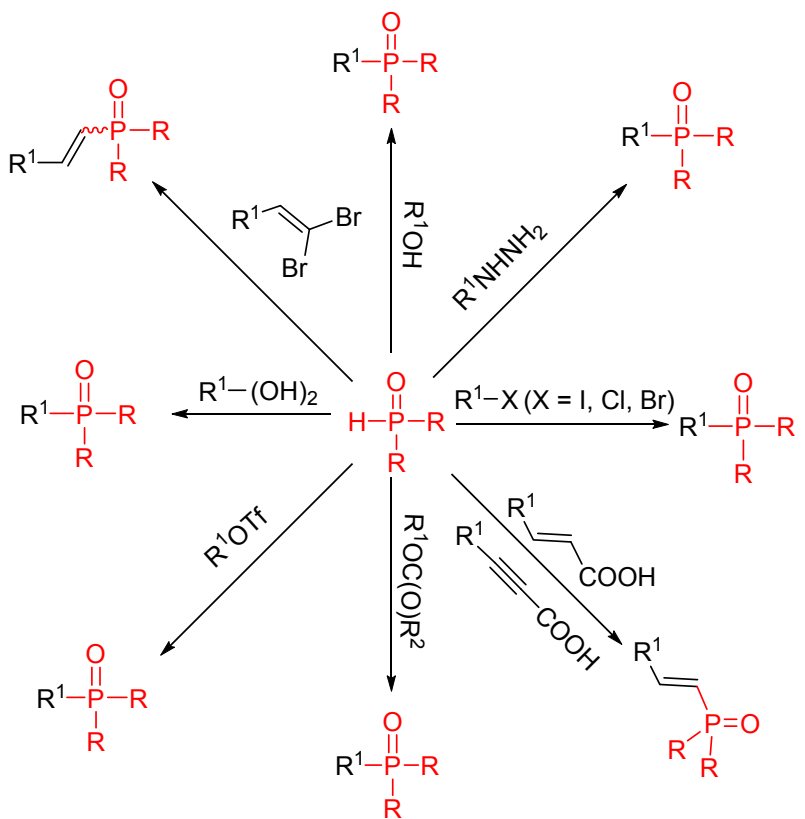

图式 2 过渡金属催化构建 $\mathrm{P}-\mathrm{C}\left(\mathrm{sp}^{2}\right)$ 键

Scheme 2 Transition metal-catalyzed coupling of $\mathrm{P}-\mathrm{C}\left(\mathrm{sp}^{2}\right)$ bond

\section{$2.1 P d$ 催化}

相对于其它过渡金属，钯催化的反应具有反应底物 适用性强、反应条件温和等特点. 目前, 绝大多数的偶 联反应都采用 $\mathrm{Pd}$ 盐作为催化剂, 尤其是在构建 $\mathrm{P}$ $\mathrm{C}\left(\mathrm{sp}^{2}\right)$ 键化合物领域, $\mathrm{Pd}$ 催化 $\mathrm{P}(\mathrm{O})-\mathrm{H}$ 键与卤代芳香化 合物之间的偶联反应是研究得较为深入的一个方向. 1980 年, Hirao 等 ${ }^{[28 ~ 30]}$ 首先采用 $\mathrm{Pd}\left(\mathrm{PPh}_{3}\right)_{4}$ 催化体系成功 实现了卤代芳烃与 $\mathrm{P}(\mathrm{O})-\mathrm{H}$ 键化合物之间的偶联(Eq. 17).

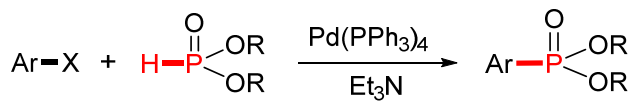

在此基础之上, 2007 年, Stawinski 等 ${ }^{[31]}$ 以 $\mathrm{Pd}(\mathrm{OAc})_{2}$ 作为催化剂，通过醋酸根离子的协同作用，实现了芳基 卤代化合物与 $\mathrm{P}(\mathrm{O})-\mathrm{H}$ 键的偶联反应(Scheme 3). 在对 反应机理的研究中发现, 该反应经历了 $\operatorname{Pd}(0)$ 的氧化加 成以及 $\operatorname{Pd}(\mathrm{II})$ 的还原消除历程，从而促进了整个偶联反 应的循环进行.

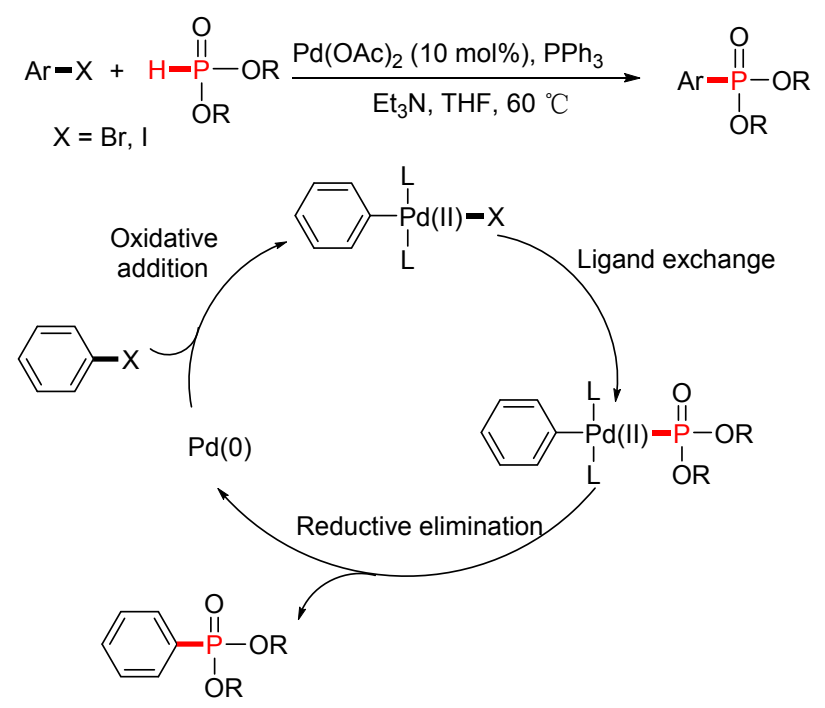

图式 $3 \mathrm{Pd}$ 催化的 P-芳基化反应

Scheme 3 Pd-catalyzed P-arylation

在 Hirao 等的研究工作发表之后, Pd 催化卤代芳烃 的 $\mathrm{P}$ 芳基化反应受到广泛关注，主要研究工作集中在反 应底物及催化体系的拓展，如反应的亲核磷试剂由亚磷 酸二烷基酯扩展到亚膦酸酯、次磷酸酯等各种 $\mathrm{P}(\mathrm{O})-\mathrm{H}$ 试剂，包括各种钯盐催化体系及反应条件也被系统考 察 ${ }^{[32 \sim 60]}$.

值得注意的是，长期以来与 $\mathrm{P}(\mathrm{O})-\mathrm{H}$ 化合物偶联的 卤代芳烃仅局限于溴、碘代物，而对于 $\mathrm{C}\left(\mathrm{sp}^{2}\right)-\mathrm{Cl}$ 键的 活化一直未能实现. 2011 年, Montchamp 等 ${ }^{[61]}$ 报道了金 属钯催化卤代芳香体系与亚磷(膦)酸酯的偶联反应，在 
膦配体的辅助作用下，首次实现了芳基氯代物与磷原子 的交叉偶联，产率为 $47 \% \sim 86 \%$ (Eq. 18).

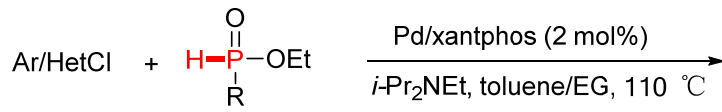

$$
\begin{aligned}
& \begin{aligned}
\text { Het/Ar }=\stackrel{\text { PI }}{\text { I }} \text { OEt } \\
\text { OEt }
\end{aligned}
\end{aligned}
$$

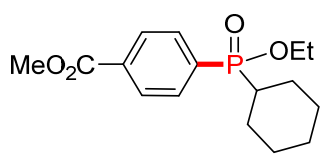

$86 \%$

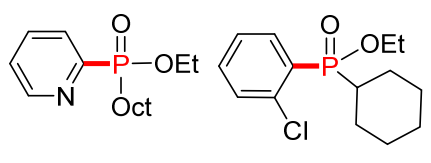

$\Delta 7 \%$
随着对反应机理的进一步认识, 关于金属钯催化 $\mathrm{P}-\mathrm{C}\left(\mathrm{sp}^{2}\right)$ 键的反应得到了更深入的研究，通过对反应 底物的拓展，已有实验表明除卤代芳烃之外，芳基硼酸 类底物也适用于此类交叉偶联反应. 2009 年，Larhed 等 ${ }^{[62]}$ 以芳基嗍酸化合物替代卤代芳烃与 $\mathrm{P}(\mathrm{O})-\mathrm{H}$ 键反 应, 微波辅助钯催化 $30 \mathrm{~min}$ 即可实现 $\mathrm{P}$ 芳基化反应, 极 大地缩短了反应时间(Eq. 19).

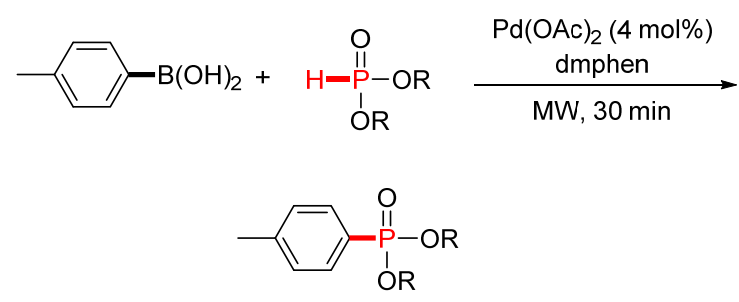

在通过缩短反应时间提高反应效率的同时，原子经 济性的提高也成为构建 $\mathrm{P}-\mathrm{C}\left(\mathrm{sp}^{2}\right)$ 键偶联反应的主要目 标. 随着钯催化脱氢交叉偶联反应的研究深入, 2012 年, 李福伟等 ${ }^{[63]}$ 报道了醋酸钯催化咪唑类化合物与亚磷酸 酯的氧化偶联反应. 在脯氨酸等配体的协同作用下，通 过对 $\mathrm{C}\left(\mathrm{sp}^{2}\right)-\mathrm{H}$ 键的活化, 从而实现了 $\mathrm{P}-\mathrm{C}\left(\mathrm{sp}^{2}\right)$ 键的高 效构建(Eq. 20). 此方法具有较高的区域选择性, 并且反 应过程中无需碱的加入，与传统的反应底物卤代芳烃相 比，此类反应的副产物为 $\mathrm{H}_{2} \mathrm{O}$, 这为合成芳基膦酸酯类 化合物提供了更为经济、有效的方法.

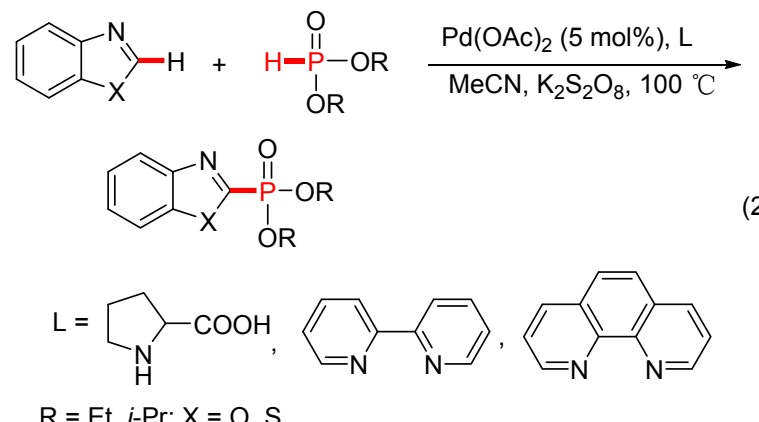

2013 年, 吴养洁课题组 ${ }^{[64]}$ 报道了氯化钯催化香豆 素与亚膦酸酯脱氢交叉偶联反应(Scheme 4), 合成了多 种天然产物香豆素的磷酰化衍生物. 通过对反应机理的 推测, $\mathrm{P}(\mathrm{O})$ - $\mathrm{H}$ 亲核试剂首先进攻 $\mathrm{Pd}(\mathrm{II})$ 催化体系形成中 间体 $B$, 香豆素 C-3 位氢原子在 $\mathrm{K}_{2} \mathrm{~S}_{2} \mathrm{O}_{8}$ 的协同作用下被 氧化的同时形成中间体 $\mathbf{D}$, 在经历了还原消除得到目标 化合物的同时，二价钯催化体系继续进入下一轮催化循 环.

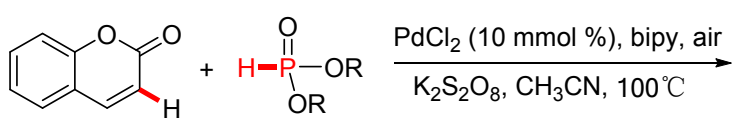

$\mathrm{R}=\mathrm{Me}, \mathrm{Et}, i-\operatorname{Pr}$ etc.<smiles>[R]OP([R])(=O)c1cc2ccccc2oc1=O</smiles>

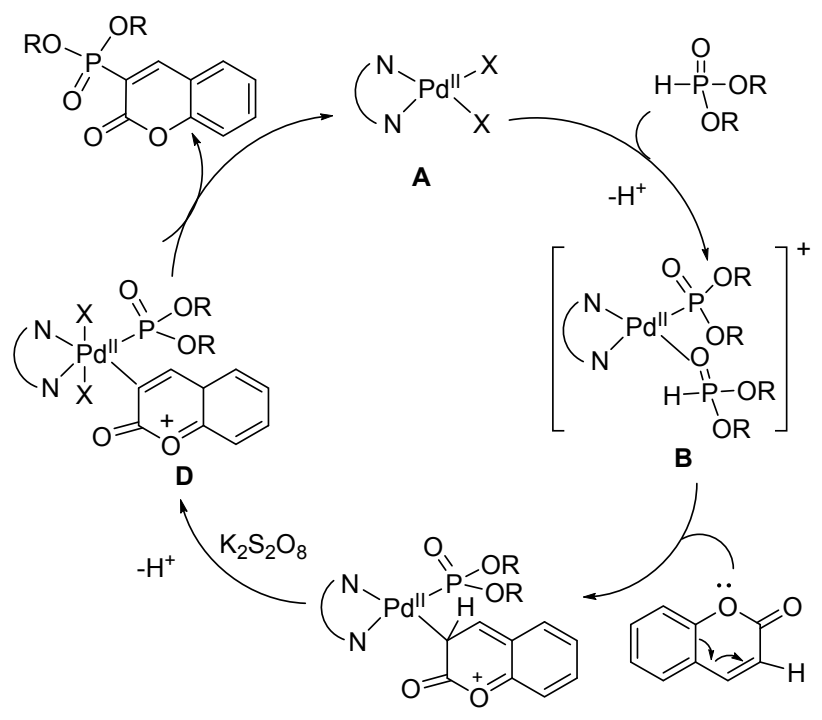

C

图式 $4 \mathrm{Pd}$ 催化脱氢偶联构建 $\mathrm{P}-\mathrm{C}\left(\mathrm{sp}^{2}\right)$ 键 Scheme $4 \mathrm{Pd}$-catalyzed $\mathrm{P}-\mathrm{C}\left(\mathrm{sp}^{2}\right)$ bond coupling via $\mathrm{CDC}$

同年，余金权等 ${ }^{[65]}$ 也以钯催化 $\mathrm{C}-\mathrm{H}$ 键活化的策略 实现了 $\mathrm{P}-\mathrm{C}\left(\mathrm{sp}^{2}\right)$ 键的构建 (Scheme 5), 通过与芳环相连 吡啶基团的导向作用，使之与催化活性中心 $\operatorname{Pd}(\mathrm{II})$ 形成 五元环过渡态，在活化 $\mathrm{C}-\mathrm{H}$ 键的同时，通过脱氢氧化 构建目标化合物 $\mathbf{3 a}$ 并释放出 $\operatorname{Pd}(0)$, 在 $\operatorname{Ag}(\mathrm{I})$ 的氧化作用 下产生的 $\operatorname{Pd}(\mathrm{II})$ 继续进入下一个催化循环.

2014 年, 赵玉芬课题组 ${ }^{[66]}$ 发展了合成 $\mathrm{P}-\mathrm{C}\left(\mathrm{sp}^{2}\right)$ 键 的新方法, 通过钯催化 $\mathrm{C}-\mathrm{N}$ 键的断裂, 实现 $\mathrm{P}-\mathrm{C}\left(\mathrm{sp}^{2}\right)$ 键的氧化偶联反应(Scheme 6). 这也是首次报道利用金 属钯催化实现芳肼与 $\mathrm{P}(\mathrm{O})-\mathrm{H}$ 键偶联形成 $\mathrm{P}-\mathrm{C}\left(\mathrm{sp}^{2}\right)$ 键 的反应，进一步丰富了 $\mathrm{Pd}$ 催化构建 $\mathrm{P}-\mathrm{C}\left(\mathrm{sp}^{2}\right)$ 键反应的 适用范围. 值得注意的是，该方法采用环境友好性的 $\mathrm{O}_{2}$ 作为氧化剂, 反应的副产物为 $\mathrm{H}_{2} \mathrm{O}$ 和 $\mathrm{N}_{2}$, 在实现高产率 目标产物转化的同时，也使反应具有良好的官能团 


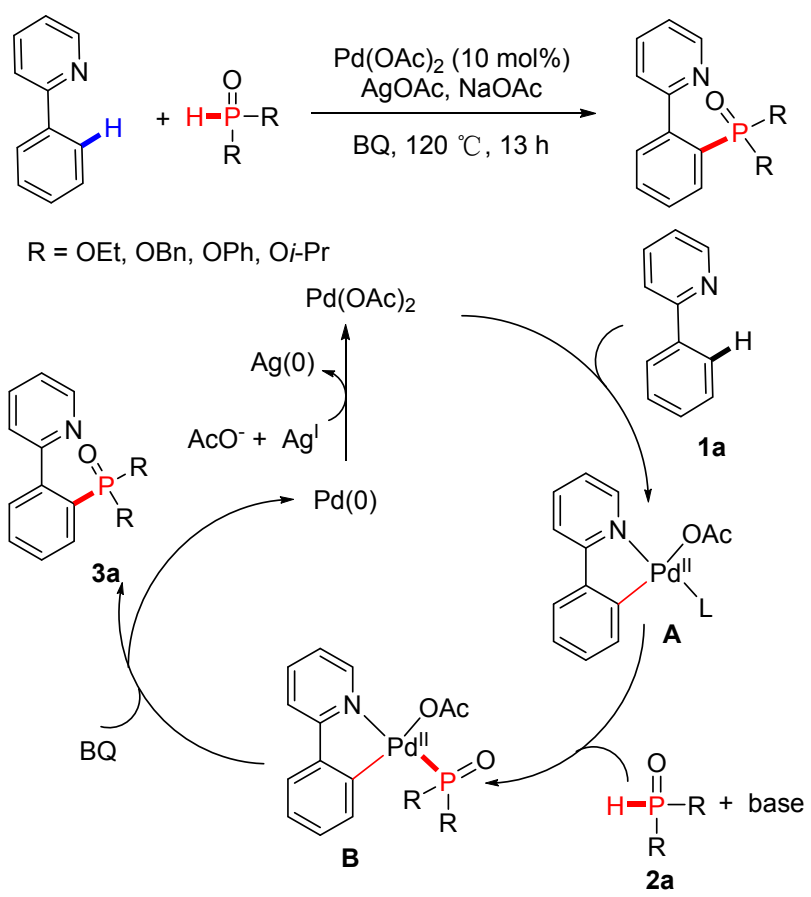

图式 $5 \mathrm{Pd}$ 催化 $\mathrm{C}-\mathrm{H}$ 活化构建 $\mathrm{P}-\mathrm{C}\left(\mathrm{sp}^{2}\right)$ 键

Scheme 5 Pd-catalyzed $\mathrm{P}-\mathrm{C}\left(\mathrm{sp}^{2}\right)$ bond coupling via $\mathrm{C}-\mathrm{H}$ activation

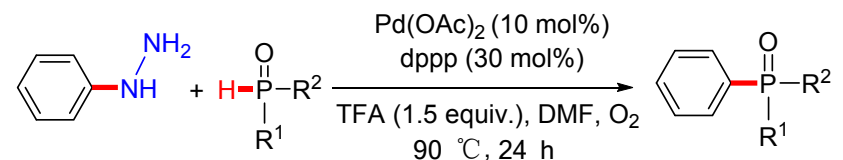

$\mathrm{R}^{1}=\mathrm{OMe}, \mathrm{OEt}, \mathrm{O} i-\mathrm{Pr}, \mathrm{Pr}, \mathrm{Ph}, n-\mathrm{Bu}$

$\mathrm{R}^{2}=\mathrm{OMe}$, OEt, Oi-Pr, $\mathrm{Pr}, \mathrm{Ph}, n-\mathrm{Bu}, \mathrm{OC}_{12} \mathrm{H}_{25}$

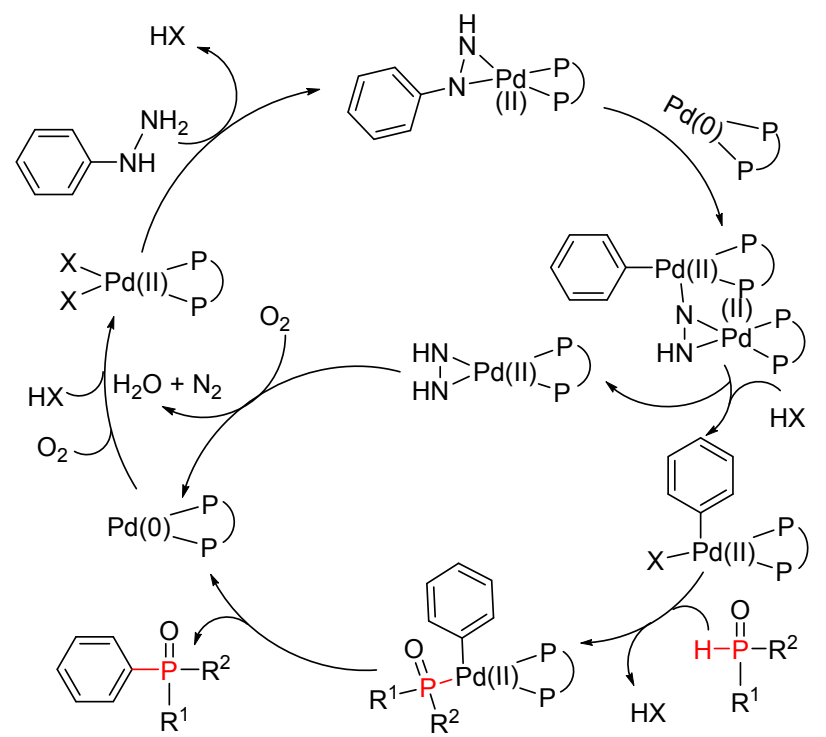

图式 $6 \mathrm{Pd}$ 催化 $\mathrm{C}-\mathrm{N}$ 活化构建 $\mathrm{P}-\mathrm{C}\left(\mathrm{sp}^{2}\right)$ 键

Scheme 6 Pd-catalyzed $\mathrm{P}-\mathrm{C}\left(\mathrm{sp}^{2}\right)$ bond formation via $\mathrm{C}-\mathrm{N}$ activation
适应性. 通过对反应机理的推测, $\mathrm{Pd}(\mathrm{II})$ 催化体系对 $\mathrm{C}\left(\mathrm{sp}^{2}\right)-\mathrm{N}$ 键的活化是整个偶联反应的决速步.

由此可见, 钯催化构建 $\mathrm{P}-\mathrm{C}\left(\mathrm{sp}^{2}\right)$ 键的偶联反应具 有条件温和、反应简单以及底物适应性强等特点, 因此 在其被发现之后的几十年里研究力度日益增加, 然而, 经过多年对钯试剂催化反应的研究与应用, 人们也逐渐 认识到了钯试剂催化反应的内在缺陷，如金属毒性较 大、价格昂贵、对不稳定且剧毒的有机膦配体具有一定 的依赖性.

\section{$2.2 \mathrm{Cu}$ 催化}

铜作为催化剂，具有价格低廉、毒性低等优点，并 且铜的化学性质比较温和, 配体简单, 正因为如此, 应 用铜盐进行反应催化是目前有机化学非常热门的一个 研究方向.

2003 年, Venkataraman 等 $^{[67]}$ 以 $\mathrm{CuI}$ 为催化剂 $(N, N-$ 二甲基乙二胺为配体), 发展了一种芳基或烯基卤代化 合物与含 $\mathrm{P}(\mathrm{O})-\mathrm{H}$ 键有机磷化合物的偶联方法, 拓展了 此类反应的催化体系，取得了较好的收率(Eq. 21). 2006 年，付华等 ${ }^{[68]}$ 对 $\mathrm{CuI}$ 催化的 $\mathrm{P}$ 芳基化反应进行改进，采 用价廉易得的脯氨酸和 2-哌啶甲酸为配体, 在很大程度 上改善了卤代芳烃与含 $\mathrm{P}(\mathrm{O})-\mathrm{H}$ 键有机磷化合物的偶 联反应(Eq. 22)

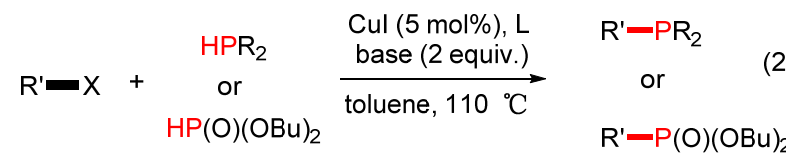

$\mathrm{R}^{\prime}=$ Aryl, Vinyl

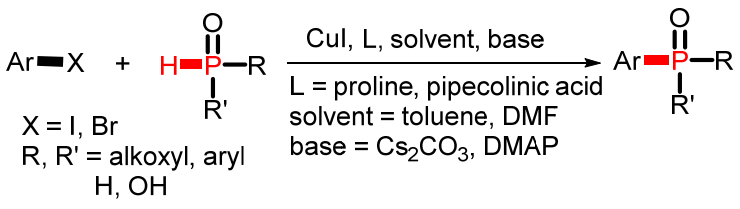

2011 年, 赵玉芬等 ${ }^{[69]}$ 以苯硼酸为底物替代卤代芳 烃，并在一价铜盐的催化下成功地实现了 $\mathrm{P}$ 芳基化反应. 该反应操作简便，室温条件下，无需惰性气体保护即可 实现高产率转化，以含氮双齿配体 1,10-菲罗啉替代含 膦配体的使用，也极大地促进了反应的进行 (Eq. 23).

2011 年, Evano 等 ${ }^{[70]}$ 以 $\mathrm{CuI}$ 为催化剂, 以 $N, N$ 二二甲 基乙二胺为配体、 $\mathrm{K}_{3} \mathrm{PO}_{4}$ 为碱，甲苯为溶剂，成功地实 现了 1,1-二澳-1-烯烃与二烷基亚磷酸酯的偶联, 得到了 高立体选择性的 $(E)-1$-烯基膦酸酯，进一步拓展了此类 反应的底物适用范围(Eq. 24).

与偶联反应过程中脱除一分子的 $\mathrm{HX}$ 相比, $\mathrm{Cu}$ 催化 脱羒偶联反应副产物仅为 $\mathrm{CO}_{2}$ 气体, 此类方法具有后处 


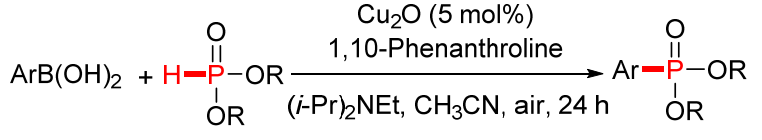

$\mathrm{R}=\mathrm{Me}, \mathrm{Et}, i-\mathrm{Pr}, \mathrm{Bn}$

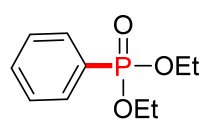

$96 \%$
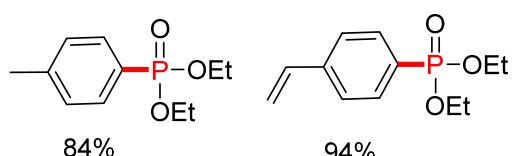

$94 \%$

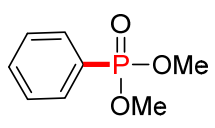

$72 \%$

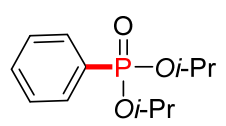

$95 \%$

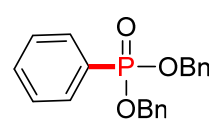

$54 \%$

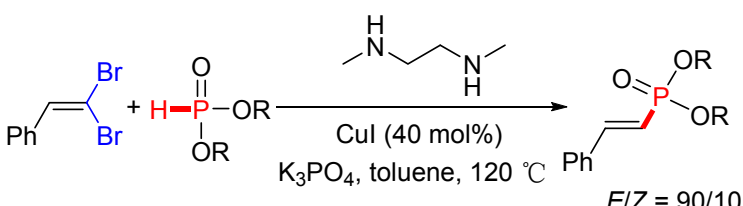

$\mathrm{R}=\mathrm{Et}, i-\mathrm{Pr}, \mathrm{Ph}, \mathrm{Bu}$

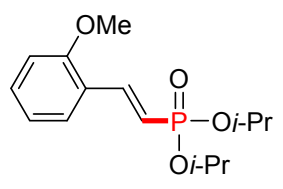

c 89/11 Yield: $67 \%$

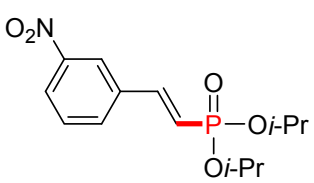

$E / Z=88 / 12$ Yield: $84 \%$<smiles>CCCOP(=O)(/C=C/c1ccc(C(F)(F)F)cc1)OCC</smiles>

$E / Z=80 / 20$ Yield: $67 \%$

理简单, 反应过程无需碱的加入等优点, 在烯基膦酸酯 类化合物的合成中得到很好的应用. 2011 年，杨尚东 等 ${ }^{[71]}$ 通过 $\mathrm{Cu}_{2} \mathrm{O} / \mathrm{Ag}_{2} \mathrm{O}$ 催化脱羧反应实现了 $\mathrm{P}-\mathrm{C}\left(\mathrm{sp}^{2}\right)$ 键 的交叉偶联(Scheme 7), 该方法可适应于不同的芳基氧 膦类化合物, 进一步拓展了 $\mathrm{P}(\mathrm{O})-\mathrm{H}$ 试剂的适用范围. 研究发现, 反应底物首先在 $\operatorname{Ag}(\mathrm{I})$ 催化下发生脱羧反应, 原位生成的 “烯基银” 进攻二苯基氧膦参与活化的 $\mathrm{Cu}$ 催化中心, 形成 $\mathrm{P}-\mathrm{C}\left(\mathrm{sp}^{2}\right)$ 键的同时释放出 $\mathrm{Cu}(\mathrm{I})$ 络合物 进入下一个催化循环. 在构建 $\mathrm{P}-\mathrm{C}\left(\mathrm{sp}^{2}\right)$ 键的过程中, 此 方法有很好的区域选择性和化学选择性.

2014 年, 赵玉芬课题组 ${ }^{[72]}$ 报道了以 $\mathrm{CuCl}$ 为催化剂 催化炔基羧酸与 $\mathrm{P}(\mathrm{O})-\mathrm{H}$ 化合物的 $\mathrm{P}-\mathrm{C}\left(\mathrm{sp}^{2}\right)$ 交叉偶联 反应. 该反应条件温和, 在氮气保护下反应 $6 \mathrm{~h}$ 即可高 产率实现 $\mathrm{P}-\mathrm{C}\left(\mathrm{sp}^{2}\right)$ 键的转化. 在无任何配体、助剂以及 碱参与的条件下, 一价铜盐参与脱羧、偶联过程, 大大 简化了此类偶联反应的操作(Eq. 25), 具有良好的应用 前景.

除此之外, 采用 “一锅法” 多组分反应策略也是高 效构建 $\mathrm{P}-\mathrm{C}\left(\mathrm{sp}^{2}\right)$ 键的又一研究方向. 2013 年, 张礼和课
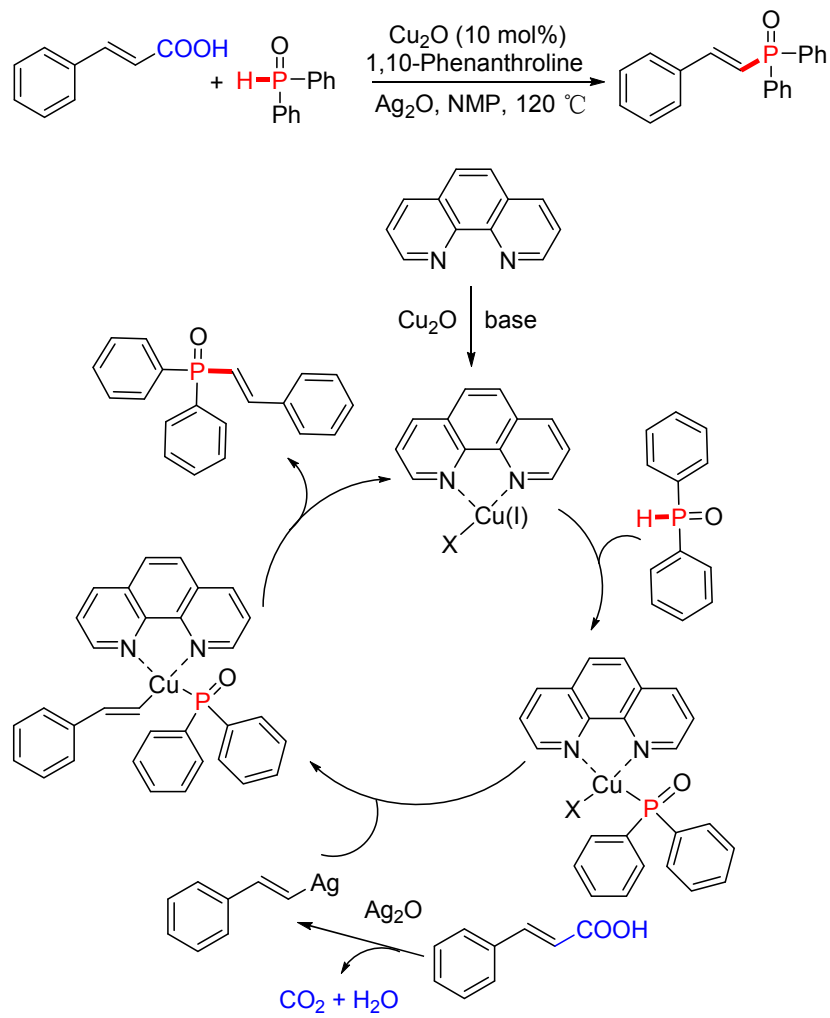

图式 $7 \mathrm{Cu}$ 催化脱羧偶联构建 $\mathrm{P}-\mathrm{C}\left(\mathrm{sp}^{2}\right)$ 键

Scheme $7 \mathrm{Cu}$-catalyzed $\mathrm{P}-\mathrm{C}\left(\mathrm{sp}^{2}\right)$ bond formation via decarboxylation-coupling

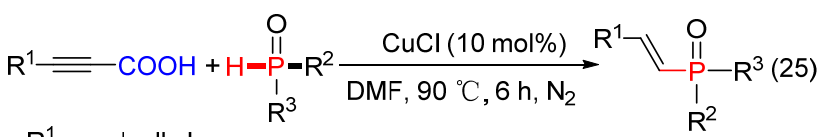

$\mathrm{R}^{1}=$ aryl, alkyl

$\mathrm{R}^{2}, \mathrm{R}^{3}=$ phenyl, ethoxy, etc.

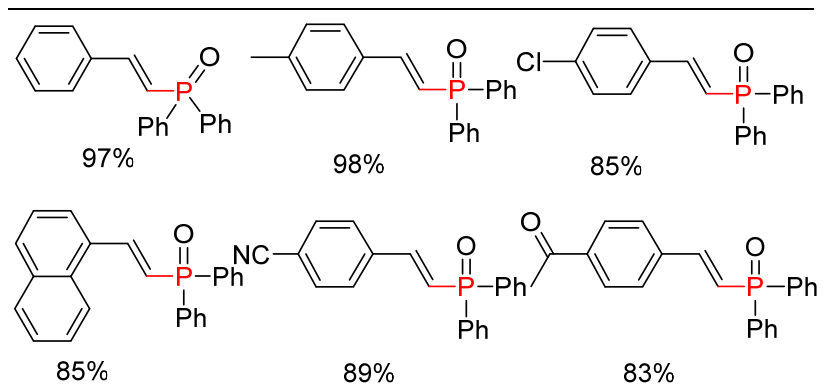

题组 ${ }^{[73]}$ 报道了 $\mathrm{CuCl}$ 催化端炔、叠氮化合物以及亚磷酸 酯类化合物的三组分偶联反应，在碱促进下，室温条件 高产率地实现了 1,2,3-三唑基-5-膦酸酯类化合物的合 成，该方法具有良好的立体选择性和基团适应性(Eq. 26).

2016 年，韩立彪等 ${ }^{[74]}$ 发展了以铜催化炔丙基醋酸 酯与 $\mathrm{P}(\mathrm{O})-\mathrm{H}$ 化合物在低温条件下的偶联反应(Eq. 27). 该反应以四甲基乙二胺(TMEDA)为配体, $N, N$-二异丙基 乙胺(DIPEA)为缚酸剂, 在乙醇溶液中 $0{ }^{\circ} \mathrm{C}$ 的条件下制 备了联烯类膦酸酯, 进一步丰富了铜盐催化 $\mathrm{P}-\mathrm{C}\left(\mathrm{sp}^{2}\right)$ 


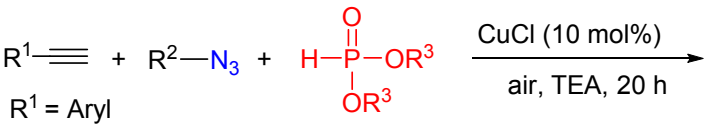

$$
\begin{aligned}
& \mathrm{R}^{2}=\mathrm{Bn}, 4-\mathrm{MeOC}_{6} \mathrm{H}_{4} \\
& \mathrm{R}^{3}=\mathrm{Et}, i-\mathrm{Pr}, \mathrm{Bn} \\
& \underset{R^{1}}{\mathrm{OR}^{2}} \stackrel{\mathrm{R}^{3}}{\mathrm{O}}
\end{aligned}
$$

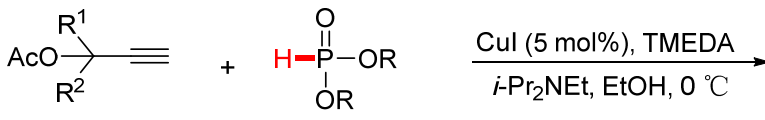

$\mathrm{R}^{1}=\mathrm{Ph}, \mathrm{Me}$

$\mathrm{R}^{2}=\mathrm{H}, \mathrm{Me}$

$$
\overbrace{\mathrm{R}^{2}}^{\mathrm{R}^{1}}==_{\mathrm{P}-\mathrm{OR}}^{\mathrm{H}}
$$

键的反应类型.

\section{$2.3 \mathrm{Ni}$ 催化}

综上所述, 从 $\mathrm{P}(\mathrm{O})-\mathrm{H}$ 键化合物出发构建 $\mathrm{P}$ $\mathrm{C}\left(\mathrm{sp}^{2}\right)$ 键的方法主要集中在钯、铜催化, 而近几年来, 过 渡金属镍参与的 $\mathrm{P}$ 芳基化反应正逐渐成为有机磷化学研 究的热点. 其主要原因是因为相对于 $\mathrm{Pd}$ 等贵金属而言, 金属 $\mathrm{Ni}$ 价廉易得, 不仅可以催化一些钯盐不能催化的 偶联反应，而且在官能团选择性以及区域选择性等方面 表现优异.

2004 年, 韩立彪等 ${ }^{[75]}$ 首次报道了室温下镍催化 $\mathrm{P}(\mathrm{O})-\mathrm{H}$ 试剂与端炔反应制备磷烯类化合物的方法, 通 过研究发现, 改变镍催化剂以及添加有机膦配体, 可以 实现 $\mathrm{P}-\mathrm{C}\left(\mathrm{sp}^{2}\right)$ 键的区域选择构建(Scheme 8).

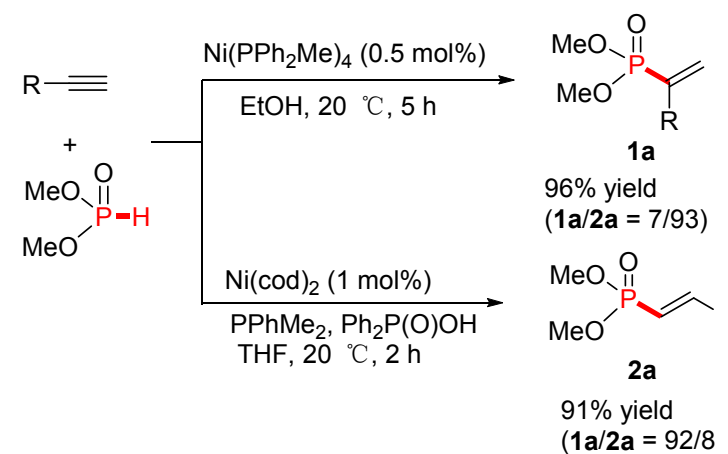

图式 $8 \mathrm{Ni}$ 催化 $\mathrm{P}(\mathrm{O})-\mathrm{H}$ 化合物与炔烃选择性构建 $\mathrm{P}-\mathrm{C}\left(\mathrm{sp}^{2}\right)$ 键

Scheme 8 Selective nickel-catalyzed addition of $\mathrm{P}(\mathrm{O})-\mathrm{H}$ bonds to alkynes

2011 年, 赵玉芬等 ${ }^{[76]}$ 报道了一种镍催化卤代芳烃 与二苯基膦氧偶联制备三芳基氧膦的新方法(Eq. 28). 该方法以价廉易得的镍盐和锌粉作为催化体系, 芳基卤 与二苯基膦氧在水中即可实现还原偶联合成三芳基膦 氧化合物. 该方法反应条件温和、操作简单、用水替代
了普遍使用的有机溶剂, 是目前合成三芳基膦氧类化合 物的绿色、经济方法.

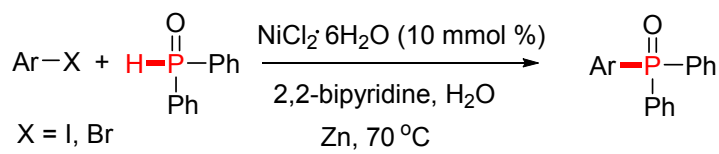

在此基础上，赵玉芬等 ${ }^{[77]}$ 对该反应体系进一步拓 展, 以 $\mathrm{NiBr}_{2}$ 为催化剂, $\mathrm{Mg}$ 为还原剂, $\mathrm{Bpy}$ 为配体, 在碱 的促进下可实现 1,1-二溴-1-烯烃与 $\mathrm{P}-\mathrm{H}$ 化合物的偶联 反应. 该方法具有很好的区域选择性以及较高的收率, 也是首次利用 $\mathrm{Ni}-\mathrm{Mg}$ 体系催化 $\mathrm{P}-\mathrm{C}\left(\mathrm{sp}^{2}\right)$ 键的合成 (Scheme 9).

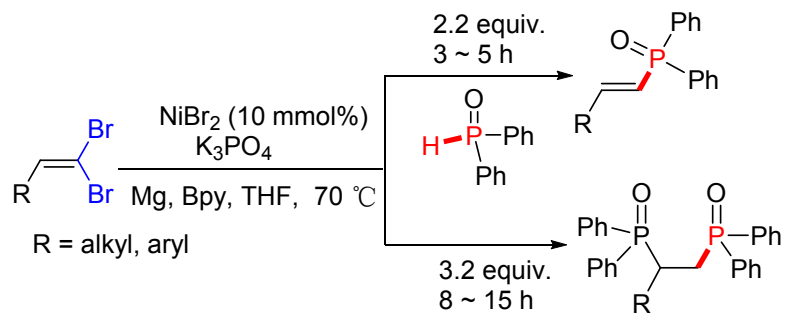

图式 $9 \mathrm{Ni}$ 催化还原偶联反应构建 $\mathrm{P}-\mathrm{C}$ 键

Scheme 9 Ni-catalyzed $\mathrm{P}-\mathrm{C}$ bond formation via reductive coupling reaction

将反应底物扩展到芳基硼酸类化合物，也是对金属 镍催化体系的进一步完善, 2013 年, 赵玉芬等 ${ }^{[78]}$ 报道了 $\mathrm{Ni}$ 催化芳基硼酸与 $\mathrm{P}(\mathrm{O})-\mathrm{H}$ 化合物的偶联反应，该反应 以 $\mathrm{NiBr}_{2}$ 为催化剂、 $\mathrm{K}_{2} \mathrm{CO}_{3}$ 为缚酸剂、吡啶为配体实现 了多种 $\mathrm{P}(\mathrm{O})$ - $\mathrm{H}$ 键与芳基喼酸化合物的高效交叉偶联, 最高收率达到 99\% (Eq. 29).

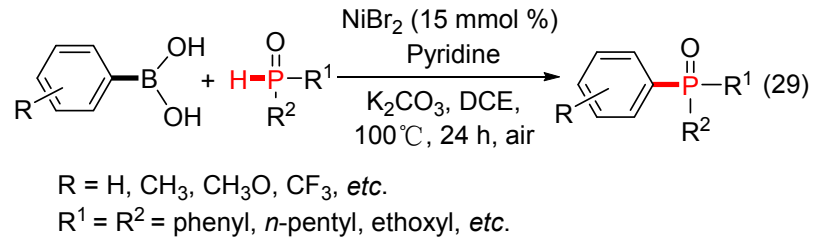

通过不同配体的选择，在改变催化活性中心活性的 同时，也实现了镍催化反应的多样性. 2014 年，赵玉芬 等 ${ }^{[79]}$ 以有机膦配体 $\mathrm{dppf}$ 代替含氮配体继续对镍催化体 系进行拓展, 在 $\operatorname{Ag}(\mathrm{I})$ 的辅助下, 成功地实现了 $\mathrm{Ni}$ 催化 脱羧交叉偶联构建 $\mathrm{P}-\mathrm{C}\left(\mathrm{sp}^{2}\right)$ 键. 该方法适用于多种不 同的 $\mathrm{P}(\mathrm{O})-\mathrm{H}$ 试剂, 并且具有很好的区域选择性. 通过 密度泛函理论(DFT)计算发现，由于膦配体具有更强的 亲和性和更大的立体空间效应，对于此类反应，相比氮 配体而言有机膦配体的参与使之具有更好的催化性能 (Eq. 30). 

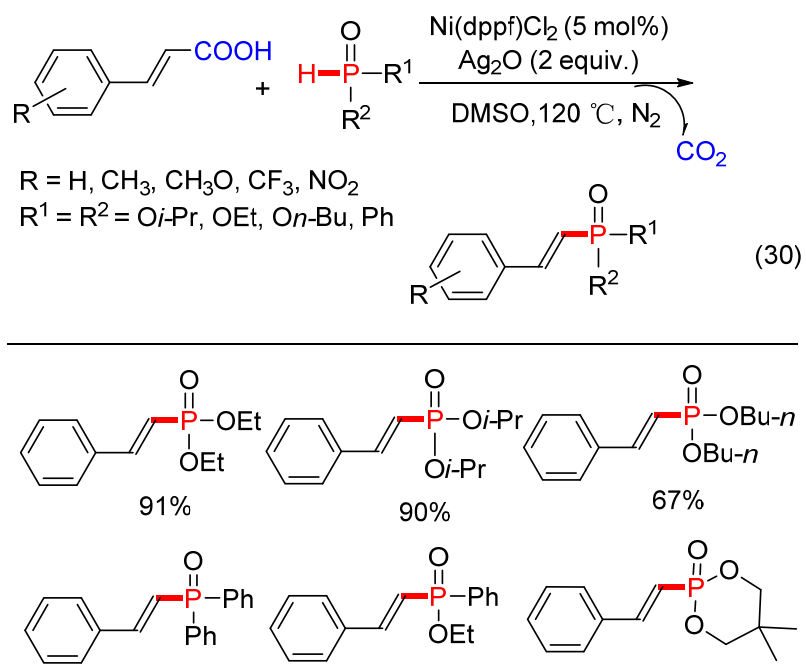

$80 \%$

$73 \%$

$69 \%$

近年来, 通过 $\mathrm{C}\left(\mathrm{sp}^{2}\right)-\mathrm{O}$ 键活化构建 $\mathrm{P}-\mathrm{C}\left(\mathrm{sp}^{2}\right)$ 键, 也是镍催化酚及其衍生物与磷试剂偶联反应的热点. 2011 年, 张万斌等 ${ }^{[80}$ 报道了镍催化亚磷酸三乙酯与芳 基三氟甲磺酸酯的类阿尔布佐夫反应. 该反应体系以 4 equiv. $\mathrm{KBr}$ 为活化试剂可以实现较高收率的芳基膦酸酯 的合成(Eq. 31).

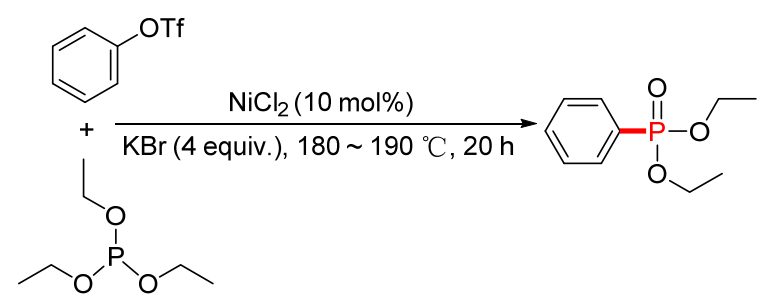

2012 年, 韩福社等 ${ }^{[81]}$ 报道了用 $\mathrm{Ni}$ 催化酚类化合物 与 $\mathrm{P}(\mathrm{O})-\mathrm{H}$ 键偶联构建 $\mathrm{P}-\mathrm{C}\left(\mathrm{sp}^{2}\right)$ 键的反应. 通过 “一锅 法” 策略, 当量三吡咯烷基溴化膦六氟磷酸盐(PyBroP) 的加入, 使得原位活化的酚类底物在 $\mathrm{NiCl}_{2}(\mathrm{dppp})$ 催化 下与多种的 $\mathrm{P}(\mathrm{O})-\mathrm{H}$ 试剂交叉偶联, 并得到了较高的产 率(Eq. 32).

2015 年, 韩立彪等 ${ }^{[82]}$ 发展了 $\mathrm{Ni}$ 催化 $\mathrm{C}-\mathrm{O}$ 键活化 构建 $\mathrm{P}-\mathrm{C}\left(\mathrm{sp}^{2}\right)$ 键的新方法. 该方法从䒺酚羧酸酯类衍 生物出发, 以 $\mathrm{Ni}(\mathrm{COD})_{2}$ 为催化剂, $\mathrm{K}_{2} \mathrm{CO}_{3}$ 为缚酸剂在较 温和条件下实现了其与 $\mathrm{P}(\mathrm{O})-\mathrm{H}$ 键的高效偶联. 反应以 $\mathrm{Ni}(0)$ 为催化活性中心, 通过氧化加成, $\mathrm{Ni}(\mathrm{II})$ 插入到 $\mathrm{C}\left(\mathrm{sp}^{2}\right)$ - $\mathrm{O}$ 键之间形成活化中间体, 经历配体交换以及 还原消除反应之后, 释放出新的零价镍进入下一个催化 循环的同时 $\mathrm{P}-\mathrm{C}\left(\mathrm{sp}^{2}\right.$ ) 键得以构建(Scheme 10).

\section{4 其他金属催化}

随着脱氢氧化偶联反应的深入研究, 2012 年, 黄志 真等 ${ }^{[83}$ 报道了银催化杂环化合物与亚磷酸酯的氧化偶
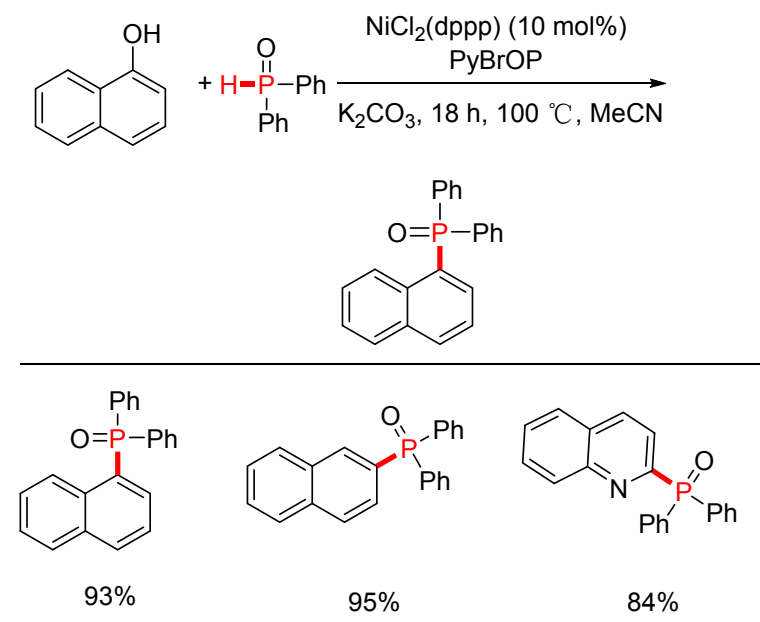

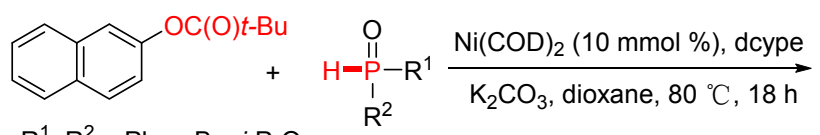
$\mathrm{R}^{1}, \mathrm{R}^{2}=\mathrm{Ph}, n-\mathrm{Bu}, i-\mathrm{PrO}$<smiles>[R]P([R])(=O)c1ccc2ccccc2c1</smiles>
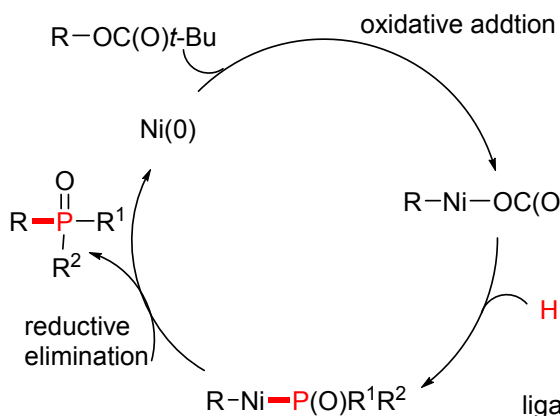

$\mathrm{R}-\mathrm{Ni}-\mathrm{OC}(\mathrm{O}) t-\mathrm{Bu}$

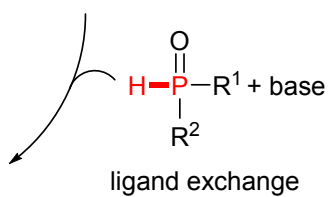

图式 $10 \mathrm{Ni}$ 催化 $\mathrm{C}-\mathrm{O}$ 活化构建 $\mathrm{P}-\mathrm{C}\left(\mathrm{sp}^{2}\right)$ 键 Scheme 10 Ni-catalyzed $\mathrm{P}-\mathrm{C}\left(\mathrm{sp}^{2}\right)$ coupling via $\mathrm{C}-\mathrm{O}$ activation

联反应(Eqs. 33, 34). 该反应以 $\mathrm{Ag}^{+} / \mathrm{S}_{2} \mathrm{O}_{8}{ }^{2-}$ 为催化体系, 通过 $\mathrm{C}\left(\mathrm{sp}^{2}\right)-\mathrm{H}$ 与 $\mathrm{P}(\mathrm{O})-\mathrm{H}$ 键的活化, 从而达到氧化脱 氢以及 $\mathrm{P}-\mathrm{C}\left(\mathrm{sp}^{2}\right)$ 键的构建. 此类方法适用于包括呋喃、 噻吩、吡咯以及吡啶衍生物在内多种杂环化合物的磷酰 化反应，并且具有底物适用广泛、反应条件温和、区域 选择性好等特点.

2015 年, 谭泽等 ${ }^{[84]}$ 报道了一种 $\mathrm{Ag}$ 催化端烯与亚磷 酸酯的脱氢偶联反应(Scheme 11). 通过自由基捕获剂 2,2,6,6-四甲基哌啶氧化物(TEMPO)的加入，很好地实 现了烯烃端位磷酰化的区域选择. 反应过程中 $\mathrm{Ag}^{+} /$ $\mathrm{S}_{2} \mathrm{O}_{8}{ }^{2-}$ 的协同作用，保证了反应体系中足量的 $\mathrm{Ag}(\mathrm{I})$ 与 $\mathrm{P}(\mathrm{O})$ - $\mathrm{H}$ 化合物作用生成磷自由基 $\mathbf{B}, \mathbf{B}$ 进攻端烯所形 成的自由基中间体 C被 TEMPO 捕获后形成化合物 D, D 经过消除反应最终得到具有良好区域选择性的目标化 合物. 


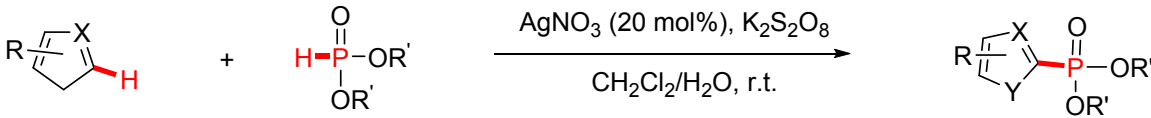

$$
\begin{aligned}
& \text { (1) } \mathrm{AgNO}_{3}(20 \mathrm{~mol} \%) \\
& \overbrace{\mathrm{H}}+\underset{\substack{\mathrm{II} \\
\mathrm{H}-\mathrm{P}_{\mathrm{O}}-\mathrm{OR}^{\prime} \\
\text { OR' }}}{\mathrm{O}} \\
& \mathrm{K}_{2} \mathrm{~S}_{2} \mathrm{O}_{8}, \mathrm{CH}_{2} \mathrm{Cl}_{2} / \mathrm{H}_{2} \mathrm{O} \text {, r.t. } \\
& \text { (2) } \mathrm{Na}_{2} \mathrm{~S}_{2} \mathrm{O}_{3} \\
& \overbrace{\mathrm{R}^{\prime} \mathrm{O}^{\prime \prime}}^{O}
\end{aligned}
$$

$\mathrm{R}^{\prime}=\mathrm{Me}, \mathrm{Et} ; \mathrm{X}=\mathrm{CH}, \mathrm{N} ; \mathrm{Y}=\mathrm{O}, \mathrm{S}, \mathrm{NMe}$.<smiles>CCOP(=O)(OCC)c1ccc(C(C)=O)o1</smiles>

$89 \%$<smiles>CCOP(=O)(OCC)c1cccs1</smiles>
$51 \%$<smiles>CCOP(=O)(OCC)c1ccc(C(C)=O)n1C</smiles>

$85 \%$<smiles>CCOP(=O)(OCC)c1ccccn1</smiles>

$81 \%$<smiles>CCOP(=O)(OCC)c1cccc(N)n1</smiles>

$70 \%$<smiles>COP(=O)(OC)c1cccc(N)n1</smiles>

$55 \%$<smiles>C=Cc1ccccc1</smiles>

$$
\frac{\underset{\mathrm{K}_{2} \mathrm{~S}_{2} \mathrm{O}_{8}}{\mathrm{AgNO}_{3}(5 \mathrm{~mol})}}{\text { TEMPO, toluene, } 100{ }^{\circ} \mathrm{C}}
$$<smiles>CCOP(=O)(/C=C/c1ccccc1)OCC</smiles>
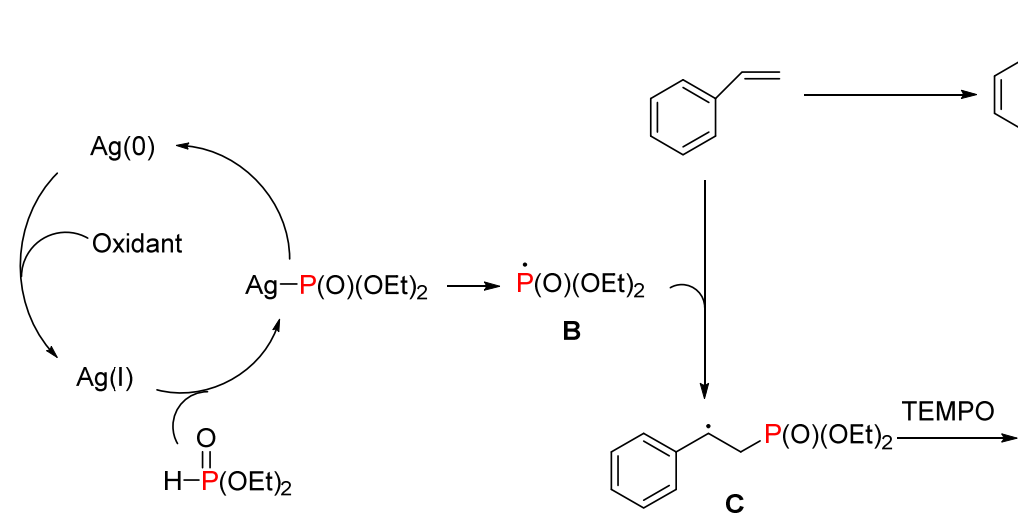<smiles>CCOP(=O)(C=Cc1ccccc1)OCC</smiles>

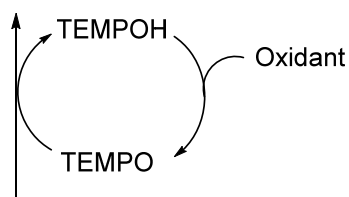

图式 $11 \mathrm{Ag}$ 催化脱氢偶联反应构建 $\mathrm{P}-\mathrm{C}\left(\mathrm{sp}^{2}\right)$

Scheme 11 Ag-catalyzed $\mathrm{P}-\mathrm{C}\left(\mathrm{sp}^{2}\right)$ coupling via $\mathrm{CDC}$

2015 年, 黄志真等 ${ }^{[85}$ 利用同样的催化体系, 对反应 底物进一步拓展, 在 $\mathrm{C}-\mathrm{H}$ 活化方向又实现新的突破 (Eq. 35). 他们利用芳香醛或酮与亚膦酸酯在 $\mathrm{Ag}^{+} /$ $\mathrm{S}_{2} \mathrm{O}_{8}{ }^{2-}$ 催化体系下的脱氢偶联反应, 高产率地合成了芳 醛或芳酮的对位磷酰化产物. 这种新型的交叉脱氢偶联 (CDC)反应具有选择性高、产率优良、官能团耐受性好 等特点.

2016 年, 赵玉芬课题组 ${ }^{[86]}$ 报道了 $\operatorname{Ag}(\mathrm{I})$ 盐催化合成 吲哚类含磷衍生物的新方法(Eq. 36), 在 $\mathrm{AgOAc}$ (3 equiv.)催化下, 芳基膦氧类 $\mathrm{P}(\mathrm{O})-\mathrm{H}$ 试剂所产生的膦自 由基进攻取代炔烃的同时, 也实现了碳氮键的构建, 但 该反应需在较高温度下进行, 产率 $28 \% \sim 74 \%$.

$\mathrm{Mn}(\mathrm{III})$ 盐作为新型的催化试剂在 $\mathrm{C}-\mathrm{H}$ 键活化、 $\mathrm{P}-\mathrm{C}$ 键偶联方面具有很重要的应用. 2010 年, 张伟、邹 建平等 ${ }^{[87]}$ 利用三价锰实现了单取代或多取代芳烃的邻、 对位磷酰化反应(Eq. 37). 在此基础之上, 他们也报道了

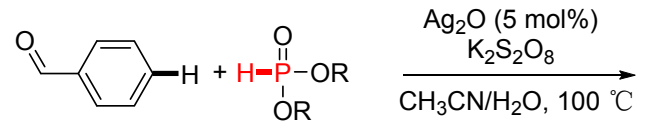

$\mathrm{R}=\mathrm{Et}, \mathrm{Me}, i-\mathrm{Pr}$

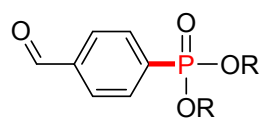

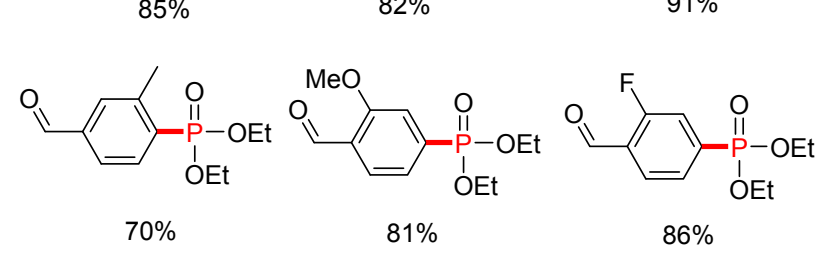

$85 \% \quad 82 \% \quad 91 \%$

$86 \%$ 
<smiles></smiles>

$\mathrm{R}^{1}=\mathrm{Ph}, \mathrm{Ar}, \mathrm{OEt} ; \mathrm{R}^{2}=\mathrm{Ph}, \mathrm{Ar}, \mathrm{OEt}$

利用相同催化体系对 $\alpha, \beta$-不饱和化合物的 $\alpha-\mathrm{H}$ 的选择性 活化, 制备了具有区域选择性的芳基烯烃磷酰化合产物 (Eq. 38). 此方法具有很好的底物适用性, 对于含有酮 基、酰胺基、硝基或酯基的底物均取得了较高的收 率 ${ }^{[88]}$.

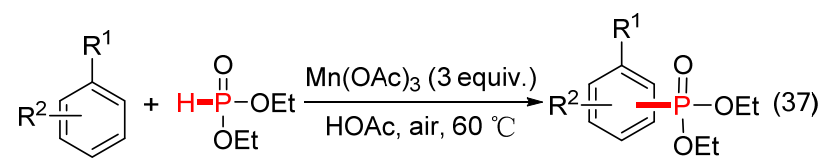

$\mathrm{R}^{1}=\mathrm{R}^{2}=$ alkyl, O-alkyl<smiles>CCOP(=O)(OCC)c1ccccc1C</smiles>

$90 \%$<smiles>CCOP(=O)(OCC)c1ccc(OC)c(OC)c1</smiles>

$95 \%$<smiles>CCOP(=O)(OCC)c1cc(C)ccc1C</smiles>

$87 \%$

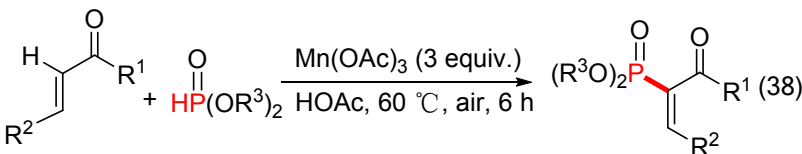

$\mathrm{R}^{1}=\mathrm{Ph}, \mathrm{Me}, \mathrm{OEt}, \mathrm{NH}_{2}$

$\mathrm{R}^{2}=\mathrm{Ph}, 4-\mathrm{MeOC}_{6} \mathrm{H}_{4}, 4-\mathrm{FC}_{6} \mathrm{H}_{6}$

$\mathrm{R}^{3}=\mathrm{Me}, \mathrm{Et}$<smiles>CO[PH](=O)C(=Cc1ccccc1)C(N)=O</smiles>

$75 \%$
$64 \%$
$77 \%$

\section{3 过渡金属催化构建 $\mathrm{P}-\mathrm{C}(\mathrm{sp})$ 键}

2000 年, Hayes 研究小组 ${ }^{[89}$ 报道了以醋酸钯、dppf、 环氧丙烷为催化体系, 以 1,1-二溴-1-烯烃、氢亚磷酸酯 为原料构建 $\mathrm{P}-\mathrm{C}(\mathrm{sp})$ 键的方法(Eq. 39), 成功制备了炔 基磷酸酯类化合物.

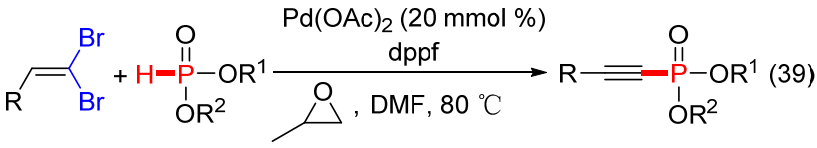

$\mathrm{R}^{1}=$ heptyl, $\mathrm{Cy}, \mathrm{Ph}, 4-\mathrm{MeC}_{6} \mathrm{H}_{4}, 4-\mathrm{MeC}(\mathrm{O}) \mathrm{C}_{6} \mathrm{H}_{4}$ $\mathrm{R}^{2}=\mathrm{Me}, \mathrm{Et}$

2011 年, Gaumont 等 ${ }^{[90]}$ 报道了在使用 $\mathrm{CuI}$ 作催化剂
的碱性条件下催化合成炔基磷嗍烷的反应(Eq. 40), 并 且得到了 $50 \% \sim 74 \%$ 的产率.

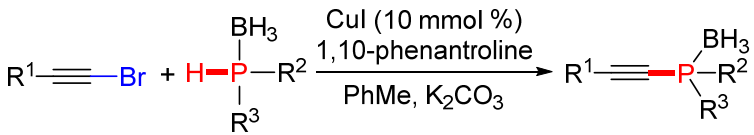

$\mathrm{R}^{1}=$ alkyl, aryl, O-aryl, $i-\mathrm{Pr}_{3} \mathrm{Si}$

$\mathrm{R}^{2}, \mathrm{R}^{3}=\mathrm{Me}, \mathrm{Et}, \mathrm{Ph}$

2009 年, 赵玉芬等 ${ }^{[91]}$ 采用脱氢偶联的方法, 在空气 氧化下实现了由简单稳定的氢亚磷酸酯与端炔偶联获 得 1-炔基膦酸酯, 并且氢亚磷酸酯可以定量转化(Eq 41). 该方法反应条件温和、操作简单、绿色经济(副产 物为水)、底物适用性广 (对含卤代基、羟基、氧基、酯 基、羰基、烯键、酰胺和磺酰胺等底物都适合)，是目前 合成 1-炔基膦酸酯的最简单、经济有效的方法，有着广 阔的应用前景

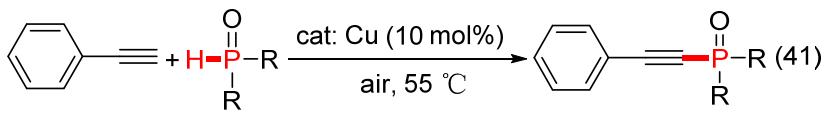

$\mathrm{R}=\mathrm{OEt}, \mathrm{O}-\mathrm{Pr}, \mathrm{O} n-\mathrm{Bu}, \mathrm{OCH}_{2} \mathrm{Ph}$

2011 年，王䂞等 ${ }^{[92]}$ 对此类反应进行了改进，使用固 定相铜盐作为催化剂合成炔基磷酸酯，室温条件下 $\mathrm{P}(\mathrm{O})-\mathrm{H}$ 试剂即可与端炔化合物发生脱氢偶联(Eq. 42), 反应以空气中的氧气作为氧化剂, 并且避免了反应体系 中碱的使用，大大简化了反应的操作。值得注意的是, 固定相铜催化剂连续使用六次以后仍具有很好的催化 活性.

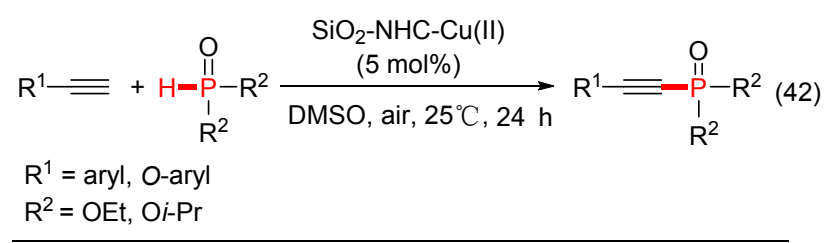

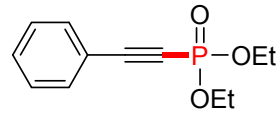

$90 \%$

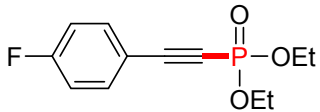

$68 \%$

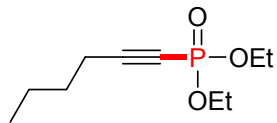

$95 \%$
2012 年, 赵玉芬课题组 ${ }^{[93]}$ 改变了铜盐的种类，以价 廉易得的 $\mathrm{CuSO}_{4} \cdot 5 \mathrm{H}_{2} \mathrm{O}$ 为催化剂, 在较温和的条件下就 可以实现端炔与亚膦酸酯的偶联反应. 经过对反应条件 的优化篎选, 确定以三乙胺 (TEA) 为碱, 二甲亚砜 (DMSO)为溶剂在 $55{ }^{\circ} \mathrm{C}$ 的条件下合成出产率优良的炔 基膦酸酯类化合物(Eq. 43). 但是，该方法仅适用于芳基 
炔丙醚类底物，具有一定的局限性.

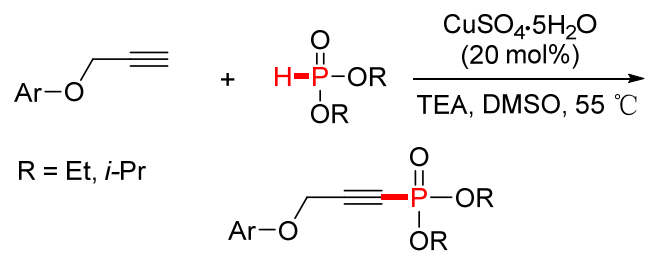

2014 年, 赵玉芬课题组 ${ }^{[94]}$ 继续对铜催化下端炔与 $\mathrm{P}(\mathrm{O})$ - $\mathrm{H}$ 键的反应机理研究(Scheme 12). 通过实验进一 步证明了空气中氧气的参与是构建磷炔 $[\mathrm{P}-\mathrm{C}(\mathrm{sp})]$ 键的 关键, 氧气的存在促进了端炔中 $\mathrm{C}(\mathrm{sp})-\mathrm{H}$ 与 $\mathrm{P}(\mathrm{O})-\mathrm{H}$ 键之间的脱氢氧化, 但若以惰性气体氮气替代氧气, 则 相同条件下生成了完全不同的磷烯 $\left[\mathrm{P}-\mathrm{C}\left(\mathrm{sp}^{2}\right)\right]$ 键, 属于 完全不同类型的加成反应.

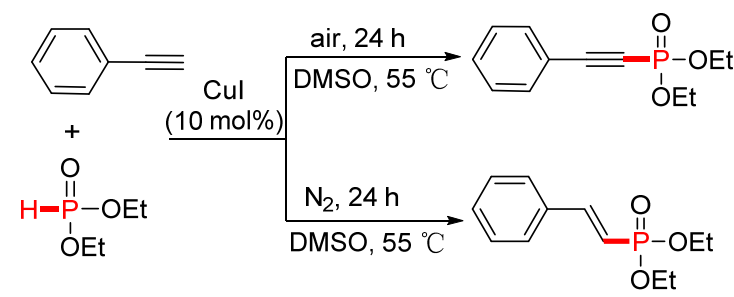

图式 $12 \mathrm{Cu}$ 催化端炔磷酰化反应的机理研究

Scheme 12 Theoretical and experimental study on $\mathrm{Cu}$-catalyzed phosphorylation of terminal alkynes

综上所述, $\mathrm{Cu}$ 盐催化磷炔键的 $\mathrm{P}-\mathrm{C}(\mathrm{sp})$ 偶联反应在 反应条件及机理方面的研究已经比较完善, 但此类反应 的 $\mathrm{P}(\mathrm{O})-\mathrm{H}$ 试剂仅适用于含有烷氧基团的亚磷酸酯类 化合物，对于芳(烷)基氧膦类底物适用性不强. 鉴于此, 2015 年, 韩立彪等 ${ }^{[95]}$ 发展了一种钯催化端炔与二苯基 氧膦的脱氢偶联反应(Scheme 13). 在温和的条件下, 以
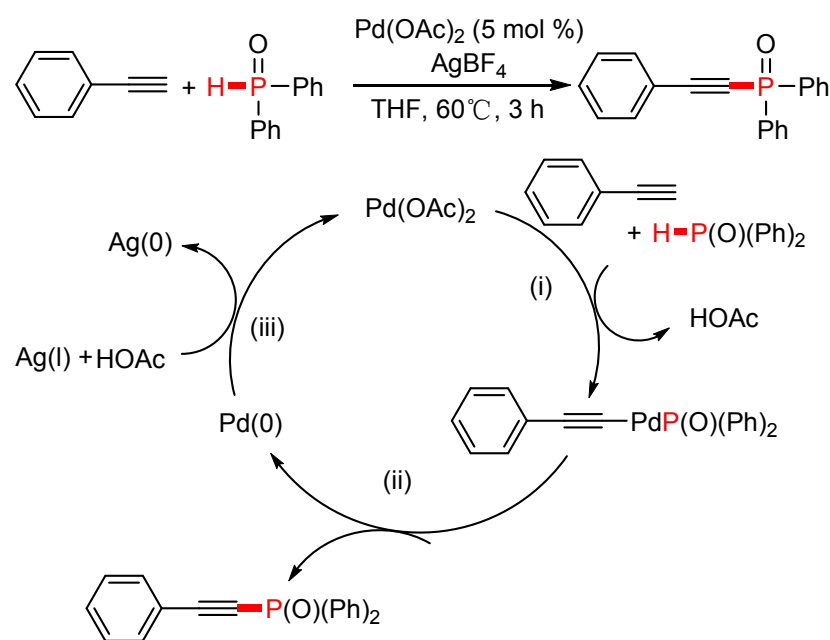

图式 $13 \mathrm{Pd}$ 催化脱氢偶联构建 $\mathrm{P}-\mathrm{C}(\mathrm{sp})$ 键 Scheme 13 Pd-catalyzed $\mathrm{P}-\mathrm{C}(\mathrm{sp})$ coupling via $\mathrm{CDC}$
醋酸钯为催化剂, 通过添加过量的 $\mathrm{AgBF}_{4}$, 从而实现了 $\mathrm{P}-\mathrm{C}(\mathrm{sp})$ 键的转化，产率高达 $99 \%$. 在反应过程中， $\operatorname{Ag}(\mathrm{I})$ 对还原消除过程中产生的 $\operatorname{Pd}(0)$ 进行氧化, 保证了 反应体系中 $\mathrm{Pd}(\mathrm{II})$ 的催化循环, 这种方法可以将不同的 $\mathrm{P}(\mathrm{O})-\mathrm{H}$ 试剂与端炔进行偶联，弥补了铜催化此类反应 的不足.

同年，雷爱文课题组 ${ }^{[96]}$ 也报道了一种新颖的银促 进端炔与 $\mathrm{P}(\mathrm{O})-\mathrm{H}$ 试剂的氧化偶联反应(Scheme 14). 反 应中过量的 $\mathrm{Ag}(\mathrm{I})$ 选择性氧化 $\mathrm{C}-\mathrm{H} 、 \mathrm{P}-\mathrm{H}$ 键形成 $\mathrm{C}-$ $\mathrm{Ag} 、 \mathrm{P}-\mathrm{Ag}$ 键, 经历中间体 $\mathbf{A}$, 并通过单电子氧化释放 $\operatorname{Ag}(0)$ 的同时生成炔基磷酸酯.

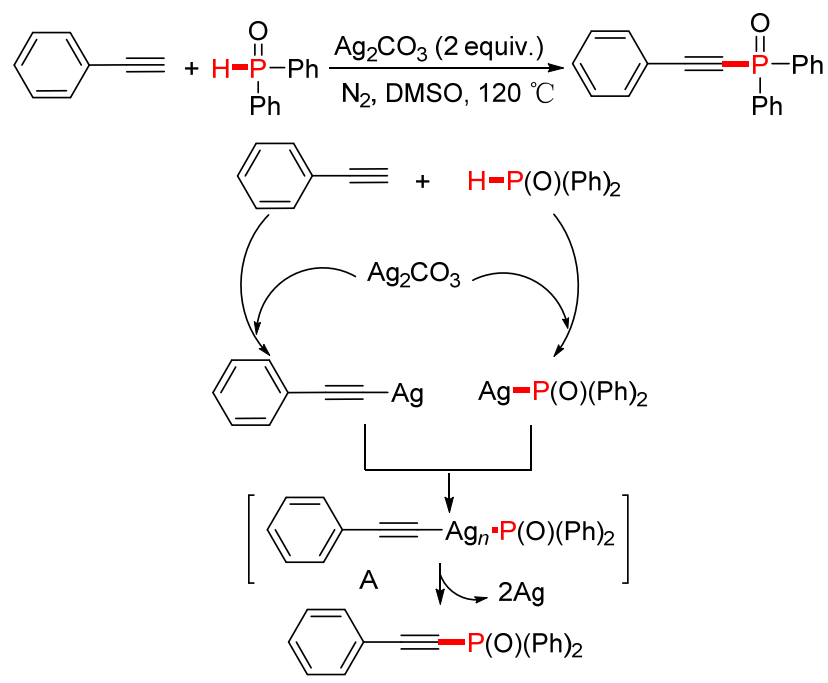

图式 $14 \mathrm{Ag}$ 催化脱氢偶联构建 $\mathrm{P}-\mathrm{C}(\mathrm{sp})$ 键

Scheme 14 Ag-catalyzed $\mathrm{P}-\mathrm{C}(\mathrm{sp})$ coupling via $\mathrm{CDC}$

吴养洁等 ${ }^{[97]}$ 继续对反应底物拓展，采用 2-甲基-3丁炔-2-醇类化合物为炔源与 $\mathrm{P}(\mathrm{O})-\mathrm{H}$ 试剂偶联, 通过 脱除丙酮进一步得到含有 $\mathrm{P}-\mathrm{C}(\mathrm{sp})$ 键的炔基磷酸酯 (Scheme 15). 反应以 $\mathrm{Pd}\left(\mathrm{PPh}_{3}\right) \mathrm{Cl}_{2}$ 为催化剂, 在缚酸剂 $\mathrm{K}_{3} \mathrm{PO}_{4}$ 的作用下脱除丙酮形成炔钯中间体，经过配体交 换, 形成 $\mathrm{P}-\mathrm{C}(\mathrm{sp})$ 键的同时释放 $\mathrm{Pd}(0), \mathrm{Ag}(\mathrm{I})$ 将其氧化成 $\operatorname{Pd}(\mathrm{II})$ 继续进入到下一催化循环.

过渡金属催化脱羧偶联反应同样也可应用于 $\mathrm{P}$ $\mathrm{C}(\mathrm{sp})$ 的构建. 2014 年, 吴玉生等 ${ }^{[98]}$ 报道了以 $\mathrm{Cu}$ 盐为催 化剂催化芳基丙炔酸与亚磷酸酯脱羧偶联合成炔基磷 酸酯的反应(Eq. 44). 该反应以 $\mathrm{Cu}(\mathrm{OAC})_{2} \cdot \mathrm{H}_{2} \mathrm{O}$ 为催化 剂, 1,10-菲罗啉为配体, 异丙醇为添加剂, 以 $\mathrm{K}_{3} \mathrm{PO}_{4}$ 为 缚酸剂，在水溶液中就可以进行。该反应具有条件温 和、绿色环保、官能团耐受性好等特点，并给出了中等 以上的产率.

\section{4 总结}

综上所述，过渡金属催化 $\mathrm{P}-\mathrm{C}$ 键的构建在近几年 

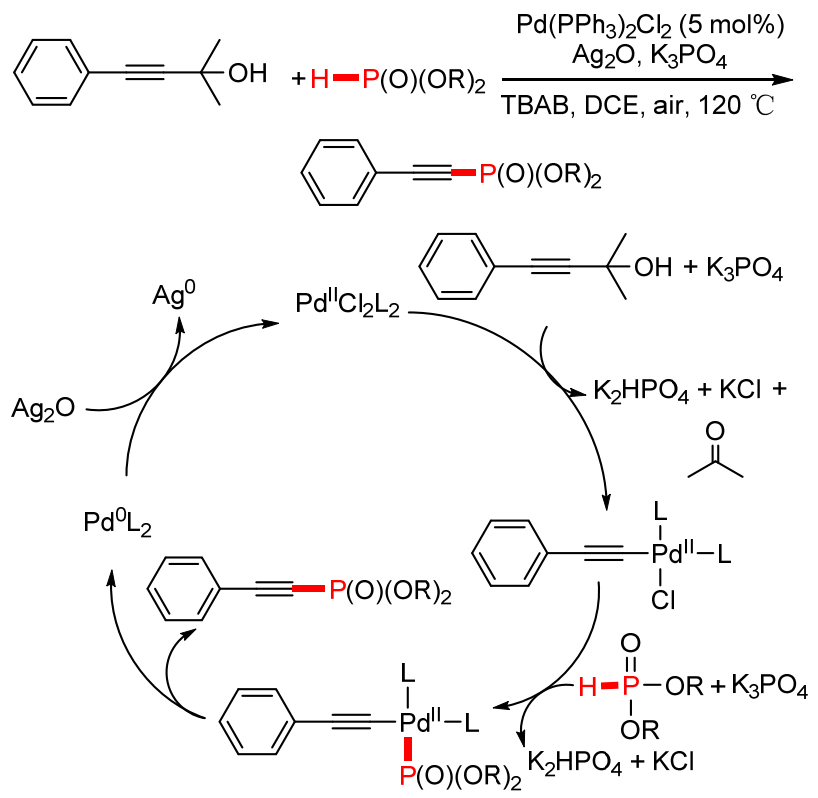

图式 $15 \mathrm{Pd} / \mathrm{Ag}$ 催化脱丙酮反应构建 $\mathrm{P}-\mathrm{C}(\mathrm{sp})$ 键

Scheme $15 \mathrm{Pd} / \mathrm{Ag}$ catalyzed $\mathrm{P}-\mathrm{C}(\mathrm{sp})$ coupling via oxidative deacetonative

$$
\mathrm{Ar}=\mathrm{COOH}+\underset{\substack{\mathrm{H}-\mathrm{P}_{\mathrm{I}}^{\mathrm{O}}-\mathrm{OR}}}{\stackrel{\mathrm{O}(\mathrm{OAc})_{2} \cdot \mathrm{H}_{2} \mathrm{O}(2 \text { equiv. })}{1,10-\text { phenanthroline }}}
$$$$
\mathrm{R}=\text { alkyl, aryl }
$$<smiles></smiles>

得到了快速发展，从传统的高温、对水和氧敏感等苛刻 反应发展到简单、高效的绿色合成，实现了巨大的飞跃， 也为有机膦化合物在光电、阻燃材料以及药物化学等研 究领域的广泛应用奠定了良好基础. 目前, 对过渡金属 催化构建 $\mathrm{P}-\mathrm{C}$ 键的研究已相对成熟, 以光催化实现 $\mathrm{C}-\mathrm{H}$ 键的活化来构建 $\mathrm{P}-\mathrm{C}$ 键已经得到逐步发展, 因其 反应条件温和, 以及较高的原子经济性等特点符合绿色 化学的发展要求, 具有广阔的发展空间.

\section{References}

[1] Baumgartner, T.; Réau, R. Chem. Rev. 2006, 106, 4681.

[2] White, A. K.; Metcalf, W. W. Annu. Rev. Microbiol. 2007, 61, 379.

[3] Metcalf, W. W.; van der Donk, W. A. Annu. Rev. Biochem. 2009, 78,65 .

[4] De Clercq, E. Med. Res. Rev. 2010, 30, 667.

[5] De Clercq, E. Med. Res. Rev. 2011, 31, 118.

[6] Dang, Q.; Kasibhatla, S. R.; Xiao, W.; Liu, Y.; DaRe, J.; Taplin, F.; Reddy, K. R.; Scarlato, G. R.; Gibson, T.; van Poelje, P. D.; Potter, S. C.; Erion, M. D. J. Med. Chem. 2010, 53, 441

[7] Maryanoff, B. E. J. Med. Chem. 2004, 47, 769.

[8] Kumar, T. S.; Zhou, S.-Y.; Joshi, B. V.; Balasubramanian, R.; Yang, T.; Liang, B.-T.; Jacobson, K. A. J. Med. Chem. 2010, 53, 2562.

[9] Kang, S.-U.; Shi, Z.-D.; Worthy, K. M.; Bindu, L. K.; Dharmawardana, P. G.; Choyke, S. J.; Bottaro, D. P.; Fisher, R. J.; Burke,
T. R., Jr. J. Med. Chem. 2005, 48, 3945.

[10] Haemers, T.; Wiesner, J.; Van Poecke, S.; Goeman, J.; Henschker, D.; Beck, E.; Jomaa, H.; Van Calenbergh, S. Bioorg. Med. Chem. Lett. 2006, 16, 1888.

[11] Seto, H.; Kuzuyama, T. Nat. Prod. Rep. 1999, 16, 589.

[12] Schwan, A. L. Chem. Soc. Rev. 2004, 33, 218.

[13] Glueck, D. S. Top. Organomet. Chem. 2010, 31, 65.

[14] Tappe, F. M. J.; Trepohl, V. T.; Oestreich, M. Synthesis 2010, 3037.

[15] (a) Rajeshwaran, G. G.; Nandakumar, M.; Sureshbabu, R.; Mohanakrishnan, A. K. Org. Lett. 2011, 13, 1270.

(b) Barney, R. J.; Richardson, R. M.; Wiemer, D. F. J. Org. Chem. 2011, 76, 2875.

[16] Cohen, R. J.; Fox, D. L.; Eubank, J. F.; Salvatore, R. N. Tetrahedron Lett. 2003, 44, 8617.

[17] Lavén, G.; Stawinski, J. Synlett 2009, 225.

[18] Baslé, O.; Li, C.-J. Chem. Commun. 2009, 4124

[19] Han, W.; Ofial, A. R. Chem. Commun. 2009, 6023.

[20] Cheng, M.-X.; Ma, R.-S.; Yang, Q.; Yang, S.-D. Org. Lett. 2016, $18,3262$.

[21] Zhang, P.-B.; Zhang, L.-L.; Gao, Y.-Z.; Xu, J.; Fang, H.; Tang, G.; Zhao, Y.-F. Chem. Commun, 2015, 51, 7839.

[22] (a) Rueping, M.; Zhu, S.-Q; Koenigs, R. M. Chem. Commun. 2011, 47, 8679 .

(b) Hari, D. P.; König, B. Org. Lett. 2011, 13, 3852.

(c) Xue, Q.-C.; Xie, J.; Jin, H.-M.; Cheng, Y.-X.; Zhu, C.-J. Org. Biomol. Chem. 2013, 11, 1606.

(d) Wang, X.-Z.; Meng, Q.-Y.; Zhong, J.-J.; Gao, X.-W.; Lei, T.; Zhao, L.-M.; Li, Z.-J.; Chen, B.; Tung, C.-H.; Wu, L.-Z. Chem. Commun. 2015, 51, 11256.

(e) Rueping, M.; Zoller, J.; Fabry, D. C.; Poscharny, K.; Koenigs, R. M.; Weirich, T. E.; Mayer, J. Chem. Eur. J. 2012, 18, 3478.

[23] (a) Xu, J.; Li, X.-Q.; Gao, Y.-Z.; Zhang, L.-L; Chen, W.-Z.; Fang, H.; Tang, G.; Zhao, Y.-F. Chem. Commun. 2015, 51, 11240.

(b) Gao, Y.-Z.; Li, X.-Q.; Xu, J.; Wu, Y.-L.; Chen, W.-Z.; Tang, G.; Zhao, Y.-F. Chem. Commun. 2015, 51, 1605.

[24] Wu, J.; Gao, Y.-Z.; Zhao, X.; Zhang, L.-L; Chen, W.-Z.; Tang, G.; Zhao, Y.-F. RSC Adv. 2016, 6, 303.

[25] (a) Tappe, F. M. J.; Trepohl, V. T.; Oestreich, M. Synthesis 2010, 3037 .

(b) Han, L.-B.; Tanaka, M. Chem. Commun. 1999, 395.

(c) Coundray, L.; Montchamp, J. Eur. J. Org. Chem. 2008, 3601.

(d) Prim, D.; Campagne, J.; Joseph, D.; Andrioletti, B. Tetrahedron 2002, 58, 2041.

(e) Schwan, A. L. Chem. Soc. Rev. 2004, 33, 218.

(f) Glueck, D. S. Synlett 2007, 2627.

[26] Seto, H.; Kuzuyama, T. Nat. Prod. Rep. 1999, 16, 589.

[27] (a) Glueck, D. S. Top. Organomet. Chem. 2010, 31, 65.

(b) Niu, M.; Fu, H.; Jiang, Y.; Zhao, Y. Chem. Commun. 2007, 272.

(c) Han, L.-B.; Tanaka, M. J. Am. Chem. Soc. 1996, 118, 1571.

(d) Han, L.-B.; Ono, Y.; Shimada, S. J. Am. Chem. Soc. 2008, 130, 2752.

[28] Hirao T.; Masunaga T.; Ohshiro Y.; Agawa. T. Tetrahedron Lett. 1980, 21, 3595.

[29] Hirao, T.; Masunaga, T.; Ohshiro, Y.; Agawa, T. Synthesis 1981, 56.

[30] Hirao T.; Masunaga T.; Yamada N, Ohshiro, Y.; Agawa, T. Bull. Chem. Soc. Jpn. 1982, 55, 909.

[31] Kalek, M.; Stawinski, J. Organometallics 2007, 26, 5840.

[32] Xu, Y.-Y.; Zhang, J. Synthesis 1984, 778

[33] Bulot, J. J.; Aboujaoude, E. E.; Collignon N.; Savignac, P. Phosphorus Sulfur Relat. Elem. 1984, 21, 197.

[34] Xu, Y.-Y.; Li, Z.; Xia, J.-Z.; Guo, H.; Huang, Y.-Z. Synthesis 
$1984,781$.

[35] Xu, Y.-Y.; Zhang, J. J. Chem. Soc., Chem. Commun. 1986, 1606.

[36] Lu, X.-Y.; Zhu, J.-Y. Synthesis 1987, 726

[37] Zhang, J.; Xu, Y.-Y.; Huang, G.-H.; Guo, H. Tetrahedron Lett. 1988, 29, 1955.

[38] Petrakis, K. S.; Nagabhushan, T. L. J. Am. Chem. Soc. 1987, 109, 2831.

[39] Holt, D.A.; Erb, J. M. Tetrahedron Lett. 1989, 30, 5393.

[40] Xu, Y.-Y.; Wei, H.-X.; Zhang, J.; Huang, G.-H. Tetrahedron Lett. 1989, 30, 949.

[41] Bennett, S. N. L.; Hall, R. G. J. Chem. Soc., Perkin Trans. 1 1995, 1145 .

[42] Trost, B. M.; Radinov, R. J. Am. Chem. Soc. 1997, 119, 5962.

[43] Machnitzki, P.; Nickel, T.; Stelzer, O.; Landgrafe, C. J. Inorg. Chem. 1998, 1029.

[44] Kazankova, M. A.; Trostyanskaya, I. G.; Lutsenko, S. V.; Beletskaya, I. P. Tetrahedron Lett 1999, 40, 569.

[45] Hall, R. G.; Riebli, P. Synlett 1999, 1633.

[46] Zhong, P.; Xiong, Z.-X.; Huang, X. Synth. Commun. 2000, 30, 273.

[47] Schuman, M.; Lopez, X.; Karplus, M.; Gouvemeur, V. Tetrahedron. 2001, 57, 10299.

[48] Kant, M.; Bischoff, S.; Siefken, R.; Gründemann, E.; Köckritz, A. J. Org. Chem. 2001, 477.

[49] Kobayashi, Y.; William, A.; Tokoro, Y. J. Org. Chem. 2001, 66, 7903.

[50] Kim, Y.-C.; Brown, S. G.; Harden, T. K.; Boyer, J. L.; Dubyak, G.; King, B. F.; Burnstock, G.; Jacobson, K. A. J. Med. Chem. 2001, 44, 340 .

[51] Cristau, H.-J.; Hervé, A.; Loiseau, F.; Virieux, D. Synthesis 2003, 2216.

[52] Xie, J.-H.; Wang, L.-X.; Fu, Y.; Zhu, S.-F.; Fan, B.-M.; Duan, H.-F.; Zhou, Q.-L. J. Am. Chem. Soc. 2003, 125, 4404.

[53] Muthukumaran, K.; Loewe, R. S.; Ambroise, A.; Tamaru, S. I.; Li, Q.; Mathur, G.; Bocian, D. F.; Misra, V. J. Org. Chem. 2004, 69, 1444.

[54] Kobayashi, Y.; William, A. D. Adv. Synth. Catal. 2004, 346, 1749.

[55] Ziessel, R. F.; Charbonnière, L. J.; Mameri, S.; Camerel, F. J. Org. Chem. 2005, 70, 9835 .

[56] Chauhan, S. S.; Varshney, A.; Verma, B.; Pennington, M. W. Tetrahedron 2007, 48, 4051.

[57] Bennett, J. A.; Hope, E. G.; Singh, K.; Stuart, A. M. J. Fluorine Chem. 2009, 130, 615.

[58] Kotek, J.; Hermann, P. L. V.; Muller, R. N.; Peters, J. A. J. Mater. Chem. 2009, 19, 1494.

[59] Ghalib, M.; Niaz, B.; Jones, P. G.; Heinicke, J. W. Tetrahedron Lett. 2012, 53, 5012 .

[60] Rankic, D. A.; Parvez, M.; Keay, B. A. Tetrahedron: Asymmetry 2012, 23, 754

[61] Deal, Er. L.; Petit, C.; Montchamp, J. L. Org. Lett. 2011, 13, 3270.

[62] Andaloussi, M.; Lindh, J.; Sävmarker, J.; Sjöberj, P. J. R.; Larhed, M. Chem. Eur. J. 2009, 15, 13069.

[63] Hou, C.-D.; Ren, Y.-L.; Lang, R.; Hu, X.-X.; Xia, C.-G.; Li, F.-W. Chem. Commun. 2012, 48, 5181

[64] Mi, X.; Huang, M.-M.; Zhang, J.-Y.; Wang, C.-Y.; Wu, Y.-J. Org. Lett. 2013, 15, 6266.

[65] Feng, C.-G.; Ye, M.-C.; Xiao, K.-J.; Li, S.-H.; Yu, J.-Q. J. $A m$ Chem. Soc. 2013, 135, 9322.

[66] Xu, W.-T.; Hu, G.-B.; Xu, P.-X.; Gao, Y.-X.; Yin, Y.-W.; Zhao, Y.-Z.
Adv. Synth. Catal. 2014, 356, 2948.

[67] Van Allen, D.; Venkataraman, D. J. Org. Chem. 2003, 68, 4590.

[68] Huang, C.; Tang, X.; Fu, H.; Jiang, Y.-Y.; Zhao, Y.-F. J. Org. Chem. 2006, 71,5020 .

[69] Zhuang, R.-Q.; Xu, J.; Cai, Z.-S.; Tang, G.; Fang, M.-J.; Zhao, Y.-F. Org. Lett. 2011, 13, 2110.

[70] Evano, G.; Tadiparthi, K.; Couty, F. Chem. Commun. 2011, 47, 179.

[71] Hu, J.; Zhao, N.; Yang, B.; Wang, G.; Guo, L.-N.; Liang, Y.-M.; Yang, S.-D. Chem. Eur. J. 2011, 17, 5516.

[72] Hu, G.-B.; Gao, Y.-X.; Zhao, Y.-F. Org. Lett. 2014, 16, 4464.

[73] Li, L.-J.; Hao, G.-L.; Zhu, A.-L.; Fan, X.-C.; Zhang, G.-S.; Zhang, L.-H. Chem. Eur. J. 2013, 19, 14403.

[74] Shen, R.-W.; Luo, B.; Yang, J.-L.; Zhang, L.-X.; Han, L.-B. Chem. Commun. 2016, 52, 6451.

[75] Han, L.-B.; Zhang, C.; Yazawa, H.; Shimada, S. J. Am. Chem. Soc. 2004, 126, 5080 .

[76] Zhang, X.-H.; Liu, H.-Z.; Hu, X.-M.; Tang, G.; Zhu, J.; Zhao, Y.-F. Org. Lett. 2011, 13, 3478.

[77] Liu, L.; Wang, Y.-L.; Zeng, Z.-P.; Xu, P.-X.; Gao, Y.-X.; Yin, Y.-W.; Zhao, Y.-F. Adv. Synth. Catal. 2013, 355, 659.

[78] Hu, G.-B.; Chen, W.-Z.; Fu, T.-T.; Peng, Z.-M.; Qiao, H.-W.; Gao, Y.-X.; Zhao, Y.-F. Org. Lett. 2013, 15, 5362.

[79] Wu, Y.-L.; Liu, L.; Yan, K.-L.; Xu, P.-X.; Gao, Y.-X.; Zhao, Y.-F. J. Org. Chem. 2014, 79, 8118.

[80] Yang, G.-Q.; Shen, C.-R.; Zhang, L.; Zhang, W.-B. Tetrahedron Lett. 2011, 52, 5032.

[81] Zhao, Y.-L.; Wu, G.-J.; Han, F.-S. Chem. Commun. 2012, 48, 5868.

[82] Yang, J.; Chen, T.-Q.; Han, L.-B. J. Am. Chem. Soc. 2015, 137, 1782.

[83] Xiang, C.-B.; Bian, Y.-J.; Mao, X.-R.; Huang, Z.-Z. J. Org. Chem. 2012, 77, 7706.

[84] Gui, Q.-W.; Hu, L.; Chen, X.; Liu, J.-D.; Tan, Z. Chem. Commun. 2015, 51, 13922.

[85] Huang, X.-F.; Wu, Q.-L.; He, J.-S.; Huang, Z.-Z. Org. Biomol. Chem. 2015, 13, 4466.

[86] Gao, Y.-Z.; Lu, G.-Z.; Zhang, P.-B.; Zhang, L.-L.; Tang, G.; Zhao, Y.-F. Org. Lett. 2016, 18, 1242.

[87] Xu, W.; Zou, J.-P.; Zhang, W. Tetrahedron Lett. 2010, 51, 2639.

[88] Pan, X.-Q.; Zou, J.-P.; Zhang, G.-L.; Zhang, W. Chem. Commun. 2010, 46, 1721.

[89] Lera, M.; Hayes, C. J. Org. Lett. 2000, 2, 3873.

[90] Bernoud, E.; Alayrac, A.; Delacroix, O.; Gaumont, A. C. Chem. Commun. 2011, 47, 3239.

[91] Gao, Y.-X.; Wang, G.; Chen, L.; Xu, P.-X; Zhao, Y.-F.; Zhou, Y.-B.; Han, L.-B. J. Am. Chem. Soc. 2009, 131, 7956.

[92] Liu, P.; Yang, J.; Li, P.-H.; Wang, L. Appl. Organometal. Chem. 2011, 25, 830 .

[93] Qu, Z.-B.; Chen, X.-L.; Yuan, J.-W.; Qu, L.-B.; Wang, F.-J.; Ding, X.-L.; Zhao, Y.-F. Can. J. Chem. 2012, 90, 747.

[94] Liu, L.; Wu, Y.-L.; Wang, Z.-S.; Zhu, J.; Zhao, Y.-F. J. Org. Chem. 2014, 79, 6816.

[95] Yang, J.; Chen, T.-Q.; Zhou, Y.-B.; Yin, S.-F.; Han, L.-B. Chem. Commun. 2015, 51, 3549.

[96] Wang, T.; Chen, S.-T.; Shao, A.-L.; Gao, M.; Huang, Y.-F.; Lei, A.-W. Org. Lett. 2015, 17, 118.

[97] Li, X.; Sun, S.-Y.; Yang, F.; Kang, J.-X.; Wu, Y.-S.; Wu, Y.-J. Org. Biomol. Chem. 2015, 13, 2432.

[98] Li, X.; Yang, F.; Wu, Y.-J.; Wu, Y.-S. Org. Lett. 2014, 16, 992. 